\title{
Collapses and watersheds in pseudomanifolds of arbitrary dimension
}

\author{
Jean Cousty, Gilles Bertrand, Michel Couprie, and Laurent Najman
}

October 2013

\begin{abstract}
This work is settled in the framework of abstract simplicial complexes. We propose a definition of a watershed and of a collapse (i.e., a homotopic retraction) for maps defined on pseudomanifolds of arbitrary dimension. Then, we establish two important results linking watersheds and homotopy. The first one generalizes a property known for distance transforms in a continuous setting to any map on pseudomanifolds: a watershed of any map is a subset of an ultimate collapse of the support of this map. The second result establishes, through an equivalence theorem, a deep link between watershed and collapse of maps: any watershed of any map can be straightforwardly obtained from an ultimate collapse of this map, and conversely any ultimate collapse of the initial map straightforwardly induces a watershed.
\end{abstract}

Keywords watershed - segmentation · collapse · topology preservation - simplicial complex . pseudomanifold

\section{Introduction}

For topographic purposes, the watershed has been extensively studied during the 19 th century $[51,14$, $41,30,11]$. One hundred years later, the watershed transform $[26,55,3,23]$ was introduced by Digabel and Lantuéjoul for image segmentation and is now used as a fundamental step in many powerful segmentation procedures [8]. Intuitively, if we see a map as a topographic surface, a watershed may be thought of as

Université Paris-Est, Laboratoire d'Informatique GaspardMonge, A3SI, ESIEE

This work received funding from the Agence Nationale de la Recherche, contract ANR-2010-BLAN-0205-03.

E-mail: \{j.cousty, g.bertrand, m.couprie, l.najman\}@esiee.fr a set of points from which a drop of water can flow down towards several distinct minima. For instance, Fig. 1a depicts a topographical relief whose watershed is made of the crests represented in black (see also the corresponding black curves in Fig. 1b).

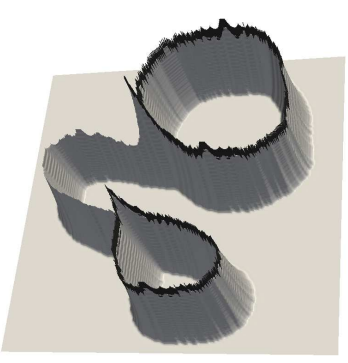

(a)

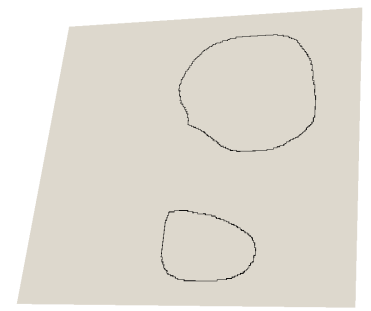

(b)
Fig. 1 Illustration of the watershed. (a): A topographical relief. (b): A watershed of (a).

The notion of topological watershed, introduced and studied in $[18,3]$, shows that the watershed transformation (i.e. the process that computes a topological watershed from a map) can be defined through a process where only one topological invariant, namely the number of connected components, is preserved.

Fig. 2a depicts an object $X$ that has two connected components. It has also two holes, meaning in $2 \mathrm{D}$, two finite connected components of the background. The number of holes is another topological invariant, that is, a quantity which is left unchanged by any continuous deformation. The intuitive notion of continuous deformation (see an example with Fig. $2 \mathrm{~b}$ and a counterexample with Fig. 2c) is formalized by the notion of homotopy (the interested reader may refer to e.g. [40] for a complete exposition). Transformations that pre- 
(a)

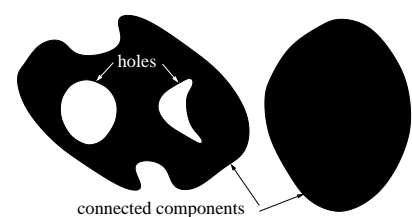

(b)

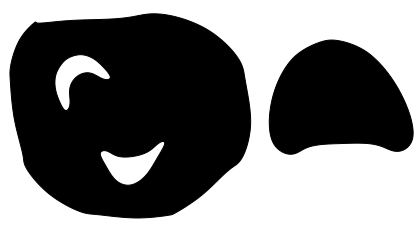

(c)

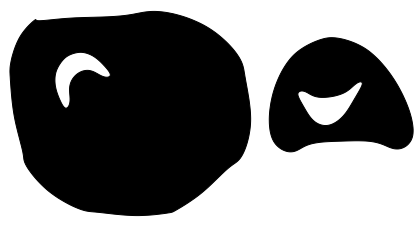

Fig. 2 Illustration of some topological invariants in $2 \mathrm{D}$. (a,b,c) are objects with 2 connected components and 2 holes, (b) can be obtained from (a) by a continuous deformation, whereas (c) cannot. Any continuous deformation preserves the topological invariants such as the number of connected components and the number of holes.

serve all topological characteristics, known as topologypreserving transformations, are used in many applications of image analysis. Homotopic skeletonization [31, $19]$ is the best known and most used transformation of this kind, with many applications both in $2 \mathrm{D}$ and $3 \mathrm{D}$. In particular, skeletons are often used as a simplification of the original data, which facilitates shape recognition, registration, or animation. In Fig. 3, we show in (b) an example of homotopic skeleton, obtained from the 2D object depicted in (a).

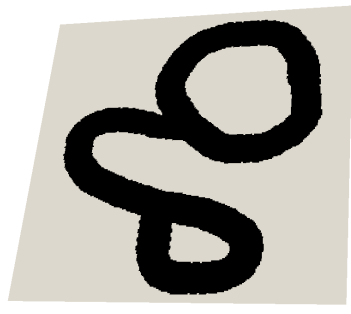

(a)

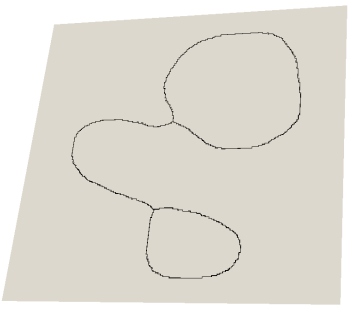

(b)
Fig. 3 Illustration of a relation between watersheds and homotopic skeletons. (a): The support of Fig. 1a. (b): A homotopic skeleton of (a).

For our purpose, it is important to mention the medial axis, a geometrical notion introduced by Blum for image analysis in the 60's [9,10]. Intuitively, the medial axis of an object $X$ is the set of those points of $X$ that have at least two closest points on the boundary of $X$ (see Fig. 4). It is thus "centered" in $X$, and in the continuous framework, it has nice topological properties which assess that the medial axis is thin and contains the same topological information as the original object. More precisely, if we consider an object $X$ that is an open subset of $\mathbb{R}^{n}$ and its medial axis $M A(X)$, then:

- there is a homotopy between $M A(X)$ and $X$, as stated by G. Matheron in [38,39], and proved by A. Rivière in $[46,47]$ and by A. Lieutier in [35].

- The interior of $M A(X)$ is empty [38,39], and moreover, $M A(X)$ is Lebesgue negligible $[46,47]$.

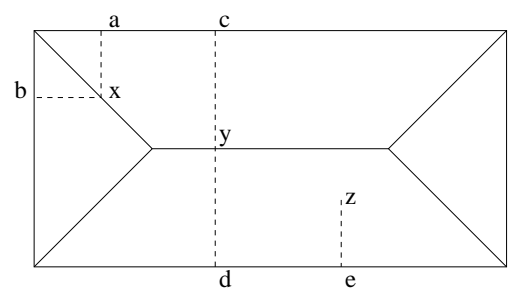

Fig. 4 Illustration of the medial axis. The object $X$ is the interior of the depicted rectangle. The points $a, b$ (resp. $c, d$ ) are the points of the boundary of $X$ closest to $x$ (resp. $y$ ), hence $x$ and $y$ are medial axis points. The point $z$ has only one closest point $e$ on the boundary, thus it is not a medial axis point. The medial axis of $X$, made of five straight line segments, is depicted.

Furthermore, if we denote by $D_{X}$ the distance map of $X$ (that is, the map that associates to each point $x$ of $X$ the Euclidean distance from $x$ to the boundary of $X$ ), the medial axis $M A(X)$ can be obtained by extracting the "crests" of $D_{X}$, or more precisely, the points $x$ of $X$ such that there exists at least two distinct steepest descent paths for $D_{X}$ starting from $x$. This property is illustrated in Fig. 5, where the distance map of a rectangle is depicted as a topographical relief, and steepest descent paths issued from two crest points are shown. Also in Fig. 1, the map depicted in (a) is indeed the distance map of the object in Fig. 3a, and the skeleton shown in Fig. 3b corresponds to a discrete notion of medial axis.

Through the notion of distance map, an interesting link between watershed and medial axis has been stated by L. Najman and M. Schmitt in [42]. They showed that the watershed of $D_{X}$ is a subset of the medial axis $M A(X)$. This property is illustrated in Figs. 1 and 3 , where it can be observed that the watershed (Fig. 1b) of the distance map (Fig. 1a) of Fig. 3a is indeed a subset of the skeleton (Fig. 3b). More precisely, the 


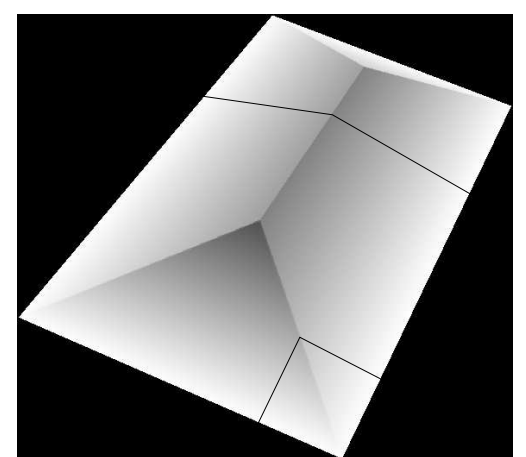

Fig. 5 Illustration of the relation between medial axis and distance map.

watershed is composed by all the points of the skeleton that are adjacent to several connected components of its complementary set.

As far as we know, such a link between watershed and medial axis had never been established in a discrete framework. Moreover, in the framework of digital topology which is used in a majority of applications in image processing, fundamental properties like homotopy and thinness of skeletons cannot be both satisfied, as shown by the counter-example of Fig. 12 .

The first main contribution of this article is a property (Theorem 34) that establishes a relation between watershed and homotopy in a discrete framework. This link is even more general than the one discussed above in the continuous framework, as it holds for arbitrary maps, distance maps being just a particular case, and for skeletons which are not necessarily medial axes. This property opens the way for defining and computing new shape descriptors that are hybrids between watersheds and skeletons.

The second main contribution of this paper is an equivalence result (Theorem 36) that establishes a deep link between watersheds and homotopy of maps, which is defined in $[7,20]$ by considering the homotopy of every level sets of the considered maps. Intuitively, it states that a set $X$ is a watershed of a map $F$ if and only if there exists a so-called ultimate skeleton $H$ of $F$ (this skeleton is also a map) such that $X$ is exactly the set of points adjacent to several distinct minima of $H$. To the best of our knowledge, no result of this kind has been obtained until now. Furthermore, due to this result, efficient algorithms based on homotopic transforms can be derived for computing a watershed of a map.

The results presented in this paper hold true in a large family of $n$-dimensional discrete spaces, namely the pseudomanifolds. This study is developed in the framework of simplicial complexes (triangulated objects) of arbitrary dimension. The notion of watershed that we use is based on the drop of water principle [23,24] and the one of homotopy relies on the collapse operation [56], a topology-preserving transformation known in algebraic topology. Additionally, this paper includes the following important contributions:

- a definition of watershed for maps defined on pseudomanifolds;

- a definition of collapse for maps defined on simplicial complexes; and

- a property of thinness of watersheds (resp. ultimate collapses) in pseudomanifolds (see Theorem 34 and Property 6 respectively): in a pseudomanifold of dimension $n$, the dimension of any watershed and of any ultimate collapse is at most $n-1$.

The proposed notions can be used for segmenting the triangulated surfaces of 3D objects (see, e.g., [44] and Fig. 6). Within this applicative context, the segmentation of simplicial complexes was the subject of many papers in the last decade. L. De Floriani et al. [28, 16] tackled the problem as a Smale-like decomposition in discrete Morse theory (see also [29]) where the simplicial complex is segmented into ascending and descending subcomplexes. Based on the same theory, H. Edelsbrunner and J. Harer [27] proposed another decomposition algorithm and they informally discuss some links with watershed algorithms. Furthermore, since the pioneering work of Mangan et al. [37], many applications involving the segmentation of 3 dimensional meshes have been developed, often without mentioning explicitly simplicial complexes. The interested reader may refer to surveys papers $[2,54]$ or to the recent SHREC'12 Track: $3 D$ mesh segmentation challenge [34]. However, as far as we know, before the present paper, a formal study of watersheds in simplicial complexes was not available.

The proposed framework can also be used for segmenting digital images equipped with triangular or cubical grids (see e.g. Fig. 7). Indeed, all notions and properties presented in this article for simplicial complexes (which include the triangular grids) can be easily transposed (see [6]) to the framework of cubical complexes (which include the cubical grids). Cubical complexes have been promoted in particular by $\mathrm{V}$. Kovalevsky [33] in order to provide a sound topological basis for image analysis. Recent advances in this framework includes the design of new image processing operators $[19,36,48]$ as well as applications in different fields such as computer graphics [43] or medical imaging $[12,13]$. 


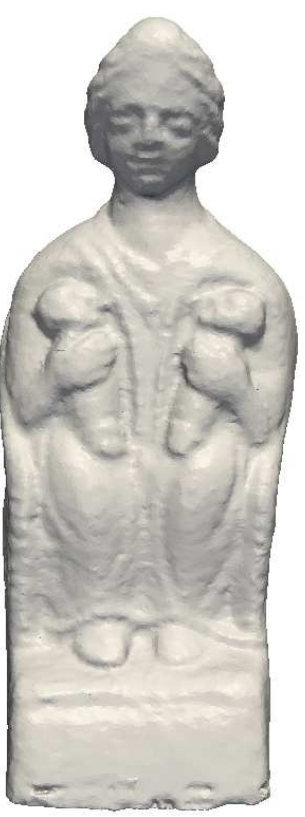

(a)

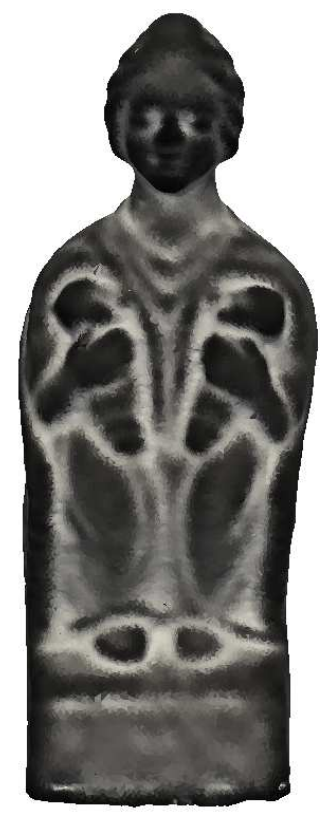

(b)

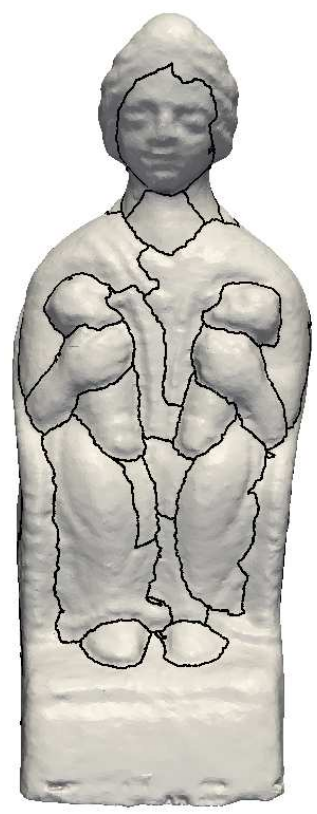

(c)

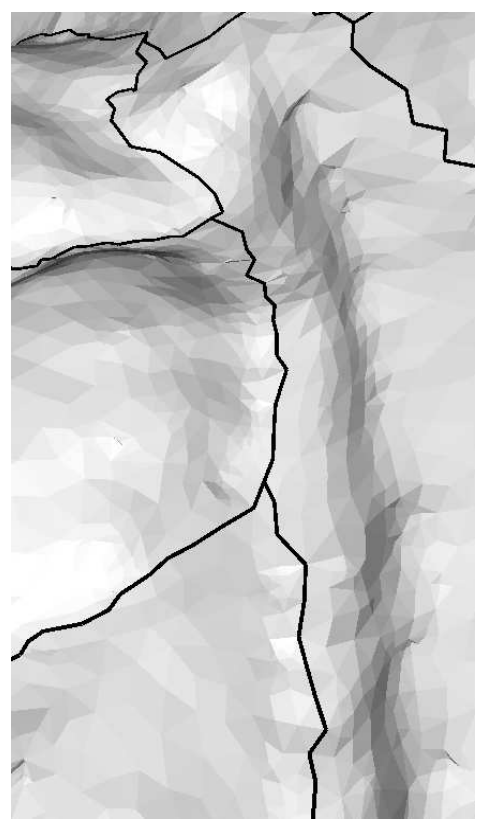

(d)

Fig. 6 (a) Rendering of a triangulated surface $\mathbb{M}$. (b) A map $F$ on $\mathbb{M}$ (which behaves like the inverse of the mean curvature of the surface, see [44]) (c) A watershed (in black) of $F$. (d) Zoom on a part of (c). The object M shown in (a) is provided by the French Museum Center for Research.

In order to ease the reading this article is selfcontained. In particular, it provides the proof of all original properties. ${ }^{1}$

\section{Simplicial complexes and pseudomanifolds}

We call (abstract) simplex any finite nonempty set. The dimension of a simplex $x$, denoted by $\operatorname{dim}(x)$, is the number of its elements minus one. In the following, a simplex of dimension $d$ will also be called a $d$-simplex. If $x$ is a simplex, we set $\operatorname{close}(x)=\{y \mid y \subseteq x, y \neq \emptyset\}$. A finite set $X$ of simplices is a cell if there exists $x \in X$ such that $X=\operatorname{close}(x)$.

Fig. 8a (resp. b, c and d) graphically represents a simplex $x$ of dimension 0 (resp. 1,2 and 3). Fig. 8e shows a cell composed of one 2-simplex, three 1simplices and three 0 -simplices.

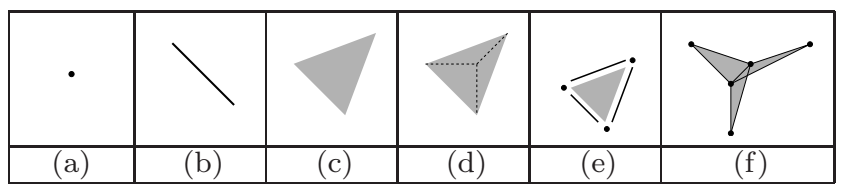

Fig. 8 (a, b, c, d) Simplices of dimension 0, 1, 2 and 3. (e) A 2-cell. (f) A complex.

\footnotetext{
1 Note that some of these properties were first presented in a conference article [22] without proof.
}

If $X$ is a finite set of simplices, we write $\operatorname{close}(X)=$ $\bigcup_{x \in X} \operatorname{close}(x)$, the set close $(X)$ is called the (simplicial) closure of $X$. A finite set $X$ of simplices is a (simplicial) complex if $X=\operatorname{close}(X)^{2}$. Let $X$ be a complex. Any element in $X$ is a face of $X$ and we call $d$ face of $X$ any face of $X$ whose dimension is $d$. Any $d$-face of $X$ that is not included in any $(d+1)$-face of $X$ is called a ( $d$-) facet of $X$. The dimension of $X$, written $\operatorname{dim}(X)$, is the largest dimension of its faces: $\operatorname{dim}(X)=\max \{\operatorname{dim}(x) \mid x \in X\}$. If $d$ is the dimension of $X$, we say that $X$ is pure whenever the dimension of all its facets equals $d$.

Intuitively, an $n$-manifold is an $n$-dimensional object that is locally "like" the $n$-dimensional Euclidean space. For instance, 2D-tori, like the one shown in Fig. 9a, are 2-manifolds whereas 2D pinched tori like those of Fig. $9 \mathrm{~b}$ and $\mathrm{c}$ are not.

The notion of $n$-pseudomanifold is less restrictive than the one of $n$-manifold. In particular, it allows "pinchings" of dimension strictly less than $n-1$ to appear. For instance, the 2D pinched torus shown in Fig. $9 \mathrm{~b}$ is a 2-pseudomanifold, contrarily to that of Fig. 9c for which a pinching of dimension 1 appears. Note that any manifold is a pseudomanifold but that

2 Note that all simplicial complexes considered in this paper are finite. Indeed, in general, the extension of the proposed notions to the case of infinite complexes is not direct and is beyond the scope of this paper. 

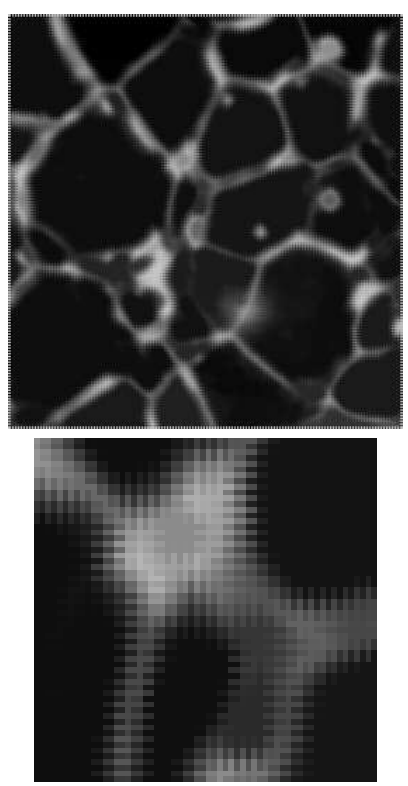

$\circ \square \circ \square \square \circ \square \circ \square 0$

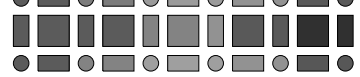
परि口卄 $0 \square 0 \square 0 \square 0 \square 0 \square 0$

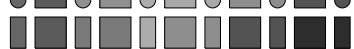
$\square \square \square$

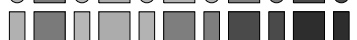
$\circ \square \circ \square \circ \square \circ \square$

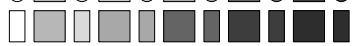
$\circ \square \circ \square \circ \square \circ \square \circ \square$ F
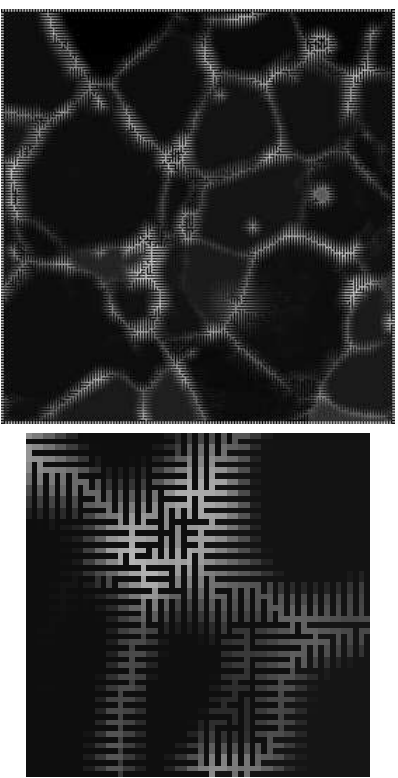

$\circ \square \square \circ \square \circ \square \circ \square \circ$

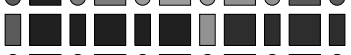

० $0 \square 0 \square 0 \square 0 \square$

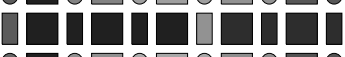

$0 \square 0 \square 0 \square 0 \square 0 \square$

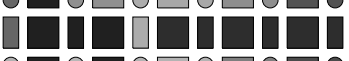
$\circ \square 00 \square 0 \square 0 \square 0$

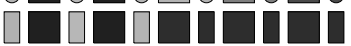
$\circ \square \square \circ \square \circ \square$ 의

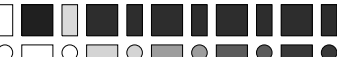

$H$
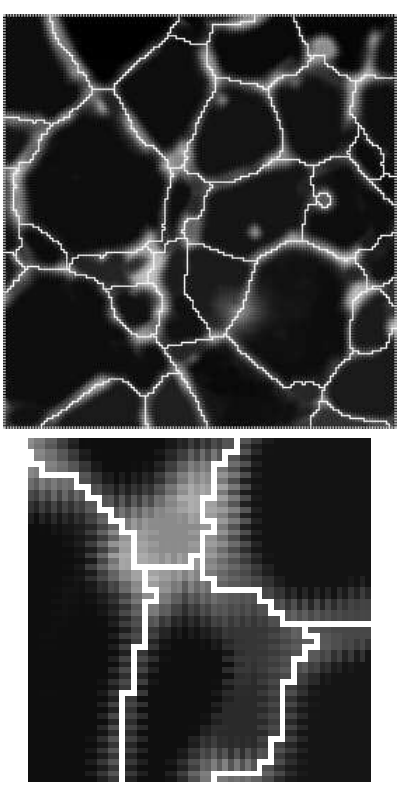

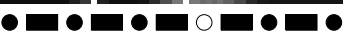

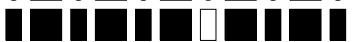

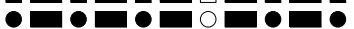
D回 $\square$ - • $\square \square \square$ - 0 D $\square$ - $10 \square 0$ 은 $\square \square \square \square \square \square \square$

$X$ (in white)

Fig. 7 A map $F$, an ultimate skeleton $H$ of $F$ and a watershed $X$ of $F$. The map $F$, defined on the two dimensional cubical complex associated to the square grid (see e.g. [5]), is obtained after some morphological filtering of an uranium oxyde image. The second (resp. third) row provides crops on a part of the images of the first (resp. second) row. The images of the third row also provide the interpretation of the images in terms of cubical complexes. As an illustration of the main result of this paper, it can be seen that the watershed $X$ of $F$ is also the set of the faces that are adjacent to two distinct regional minima of $H$.

the converse is not true. Pseudomanifolds, that are often used in computer graphics applications, constitute the class of discrete spaces studied in this paper. The notion of a pseudomanifold considered in this article implicitly refers to objects without boundary (see e.g. [40] for formal definitions of these notions). Digital images are in general defined on a (hyper) rectangular domain with boundaries. To handle a digital image in the proposed framework, one can embed its domain into a pseudomanifold without boundary. For instance, every boundary element can be linked to an additional point so that the obtained domain has the same topology as a sphere. Alternatively, the "opposite" boundaries of the domain can be identified in order to obtain a torus. In fact, the definitions and properties presented in this paper can be adapted (this would be rather technical and would increase the length of the proofs) to handle the case of objects with boundaries, allowing for handling digital images in a direct way, as done in the illustration of Fig. 7.

The remaining part of this section is devoted to the formal definition of pseudomanifolds, in the framework of simplicial complexes, and to a lemma that will be used for proving the main claims of the paper.

Let $X$ be a set of simplices, and let $d \in \mathbb{N}$. Let $\pi=$ $\left\langle x_{0}, \ldots, x_{\ell}\right\rangle$ be an ordered sequence of $d$-simplices in $X$. The sequence $\pi$ is a $d$-path from $x_{0}$ to $x_{\ell}$ in $X$ if $x_{i-1} \cap x_{i}$ is a $(d-1)$-simplex in $X$, for any $i \in\{1, \ldots, \ell\}$. Two $d$-simplices $x$ and $y$ in $X$ are said to be $d$-linked for $X$ if there exists a $d$-path from $x$ to $y$ in $X$. We say that the set $X$ is $d$-connected if any two $d$-simplices in $X$ are $d$-linked for $X$.

Let $X$ be a set of simplices, and let $\pi=\left\langle x_{0}, \ldots, x_{\ell}\right\rangle$ be a $d$-path in $X$. The $d$-path $\pi$ is said simple if for any two distinct $i$ and $j$ in $\{0, \ldots, \ell\}, x_{i} \neq x_{j}$. It can be easily seen that $X$ is $d$-connected if and only if, for 


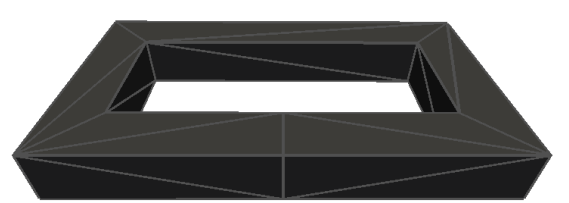

(a)

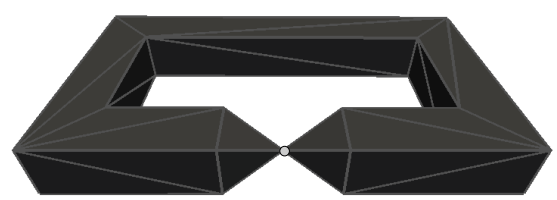

(b)

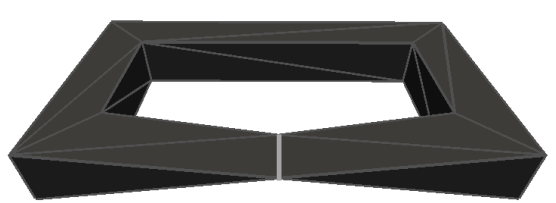

(c)

Fig. 9 (a) A topological torus. (b,c) Pinched tori: the pinchings (of dimensions 0 and 1 respectively) are marked in light gray.

any two $d$-simplices $x$ and $y$ of $X$, there exists a simple $d$-path from $x$ to $y$ in $X$.

Definition 1 A complex $X$ of dimension $d$ is a $d$ pseudomanifold if:

1. $X$ is pure; and

2. any $(d-1)$-face of $X$ is included in exactly two $d$ faces of $X$; and

3. $X$ is d-connected.

The complex of Fig. $8 \mathrm{f}$ is pure, its dimension is 2 , but it is not a 2-pseudomanifold. Indeed, it contains six 1 -faces that belong to only one 2 -face. Remark also that it cannot be a subset of a 2-pseudomanifold since it contains one 1-face that belongs to three 2-faces. Fig. 10a shows a subset of a 2-pseudomanifold.

Important notation 2 In this paper $\mathbb{M}$ stands for any $n$-pseudomanifold, where $n$ is a positive integer.

In the next sections, we will study transformations that preserve the number of connected components of the complement of complexes. In general, the complement of a complex is not a complex. Therefore, we introduce the following notions that allows the complement of complexes to be handled.

Let $x \in \mathbb{M}$, the star of $x$ (in $\mathbb{M}$ ), denoted by $\operatorname{star}(x)$, is the set of all simplices of $\mathbb{M}$ that include $x$, i.e., $\operatorname{star}(x)=\{y \in \mathbb{M} \mid x \subseteq y\}$. If $A$ is a subset of $\mathbb{M}$, the set $\operatorname{star}(A)=\cup_{x \in A} \operatorname{star}(x)$ is called the star of $A$ (in $\mathbb{M}$ ). A set $A$ of simplices of $\mathbb{M}$ is a $\operatorname{star}($ in $\mathbb{M}$ ) if $A=\operatorname{star}(A)$.

If $X \subseteq \mathbb{M}$, we denote by $\bar{X}$ the complementary set of $X$ in $\mathbb{M}$, i.e. $\bar{X}=\mathbb{M} \backslash X$.

Let $X$ be a subset of $\mathbb{M}$. Observe that, in general, if $X$ is a complex, then $\bar{X}$ is not a complex. The following property clarifies the links between the subsets of $\mathbb{M}$ and their complementary sets, and follows immediately from the definitions. Note that this property holds true even if the complex $\mathbb{M}$ is not an $n$ pseudomanifold.

Property 3 Let $X \subseteq \mathbb{M}$. The set $X$ is a complex if and only if $\bar{X}$ is a star.
The empty set and $\mathbb{M}$ are the only subsets of $\mathbb{M}$ which are both a complex and a star.

Any subset of a complex $X$ which is also a complex is called subcomplex of $X$. If $Y$ is a subcomplex of $X$, we write $Y \preceq X$. Any subset of a star $A$ which is also a star is called substar of $A$. If $B$ is a substar of $A$, we write $B \sqsubseteq A$.

Important notation 4 In this paper, we will only consider $d$-paths in $\mathbb{M}$ with $d=n$. Therefore, to shorten the notations, we will use the term path (resp. linked and connected) instead of n-path (resp. n-linked and $n$ connected).

Let $A \sqsubseteq \mathbb{M}$ and let $B \sqsubseteq A$. We say that $B$ is a (connected) component of $A$ if $B$ is a connected substar of $A$ which is maximal for this property, i.e., for any connected star $C$ in $\mathbb{M}, B \sqsubseteq C \sqsubseteq A$ implies $C=B$.

To finish this section, we introduce a property of the pseudomanifolds that will be used in the next section to establish the main claims of this paper.

Lemma 5 Let $x \in \mathbb{M}$ be an $(n-2)$-face of $\mathbb{M}$, let $y$ be an $(n-1)$-face that contains $x$, and let $x_{0}$ and $x_{1}$ be the two distinct $n$-faces of $\mathbb{M}$ that contain $y$. Then, $x_{0}$ and $x_{1}$ are linked for $\operatorname{star}(x) \backslash\{y\}$.

The proof of Lemma 5 is given in Appendix A.

Fig. 11 shows the set of 2 -faces belonging to $\operatorname{star}(x)$, where $x$ is a 0 -face of some 2-pseudomanifold. Let us consider the two 2-faces $x_{0}$ and $x_{1}$ and the 1face $y=x_{0} \cap x_{1}$, which indeed includes $x$. It can be verified that $x_{1}$ and $x_{0}$ are linked for $\operatorname{star}(x) \backslash\{y\}$ since $\left\langle x_{1}, \ldots, x_{7}, x_{0}\right\rangle$ is a path in $\operatorname{star}(x) \backslash\{y\}$.

\section{Homotopic transforms: collapses}

For applications to 2D and 3D image processing, homotopies are often tackled in the framework of digital topology [31] for which the notion of a simple pixel or point is central. Intuitively, a pixel of a $2 \mathrm{D}$ black object lying in a white background is said to be simple if its removal from the set of black pixels does not alter topology. For instance, in Fig. 12a, the pixel labeled $x$ is simple for the set $X$ drawn in black. In particular, it can 


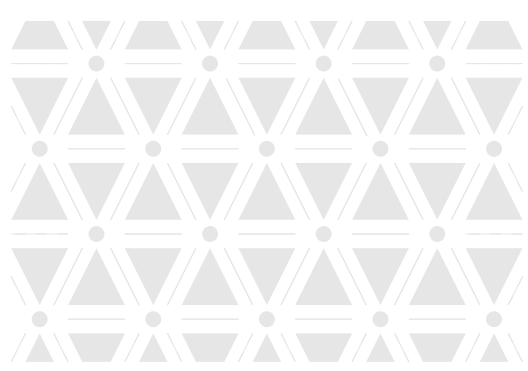

(a)

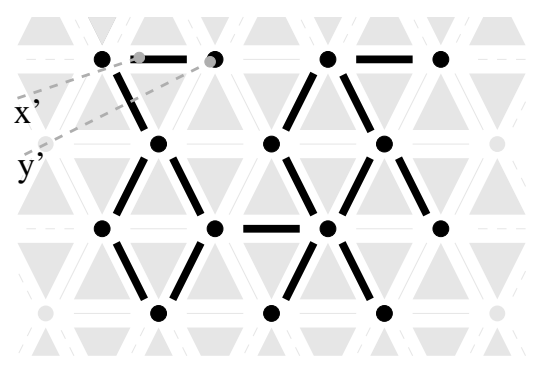

(d)

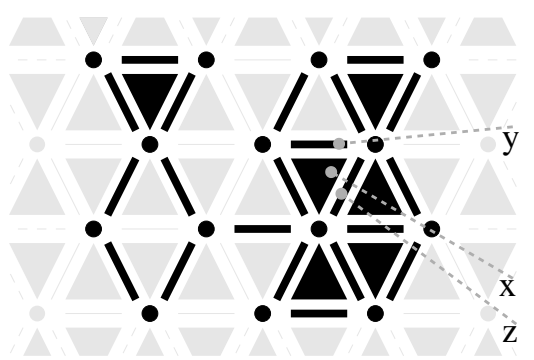

(b)

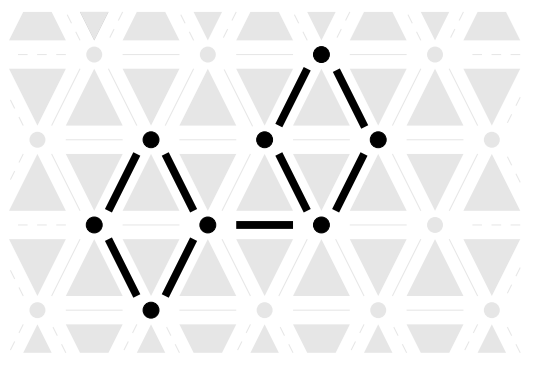

(e)

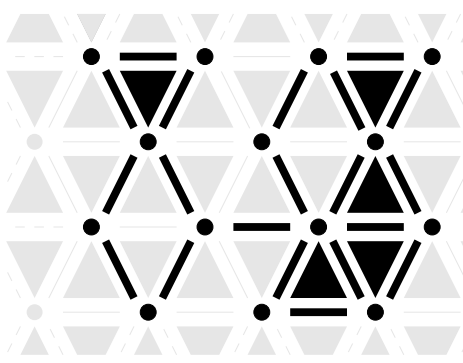

(c)

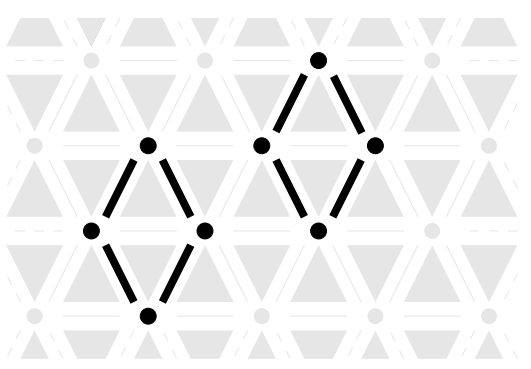

(f)

Fig. 10 (a) A subset of a 2-pseudomanifold $\mathbb{M}$. (b) A subcomplex $X$ of $\mathbb{M}$ in black. (c) An elementary collapse $Y$ of $X$. (d) An ultimate 2-collapse $Z$ of both $X$ and $Y$. (e) An ultimate 1-collapse $W$ of $Z$. (f) A cut for $\bar{X}$.

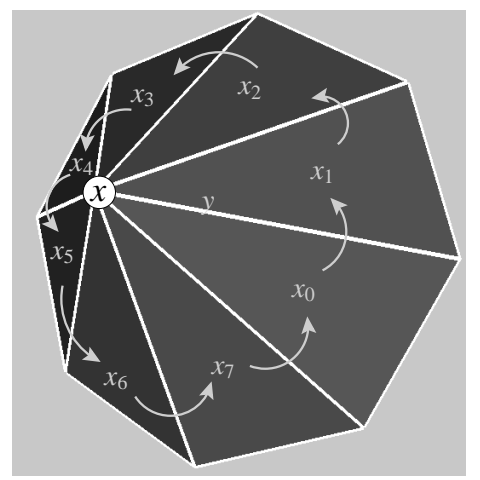

Fig. 11 Illustration of Lemma 5 and of a local cycle (Appendix A).

be verified that the sets $X$ and $X \backslash\{x\}$ have the same number of connected components and "holes". On the contrary, in Fig. 12, the removal of the pixel labeled $w$ would merge two "holes" of $X$ and therefore $w$ is not simple. The pixel $y$ (resp. $z$ ) is not simple either, since its removal would suppress (resp. create) a "hole". A set $Y$ of black pixels is a (homotopic) skeleton of an object $X$, if $Y$ may be derived from $X$ by iterative removal of simple pixels. For instance, in Fig. 12, the object $Y$ is a skeleton of $X$. It can be observed in particular that $X$ and $Y$ have the same number of connected components and "holes". In this framework of digital topology, a skeleton is said to be an ultimate skeleton if it does not contain any simple pixel, that is if it cannot be reduced, by pixel removal, while leaving topology unchanged. For instance, it can be verified that the set $Y$ (Fig. 12) does not contain any simple pixel. Thus, the set $Y$ is an ultimate skeleton of $X$.

Intuitively, one may think of an ultimate skeleton in a 2D image as a 1D object. However, it is well known in digital topology (see e.g. [1]) that an ultimate skeleton can be arbitrarily thick. For instance, in Fig. 12b, the point $z$, which belongs to the ultimate skeleton $Y$, is not adjacent to any white pixel. Thus, $Y$ is thick and cannot be considered as a 1D object. Fig. 13 shows similar examples for 2D objects in the triangular and hexagonal grids which are popular in the field of mathematical morphology (see chapter XI of [53]). Similar examples can also be found in 3D (see, for instance, the object of Fig. 15b in [21]). In practice, such thickness leads to important difficulties for post-processing skeletons.

In this section, we present the operation of collapse of a complex introduced by J.H.C. Whitehead [56], which is a discrete analogue of a retraction, that is, a continuous (homotopic) deformation of an object onto one of its subsets. As introduced in [4] (see also [32, 19]), the collapse operation allows the main notions (and results) of digital topology, including the one of simple pixel, to be retrieved. Fig. 12 shows an example of an ultimate collapse of an object in the 2D square grid. Property 6 given in this section asserts that, in an $n$-pseudomanifold, an ultimate collapse is always thin 


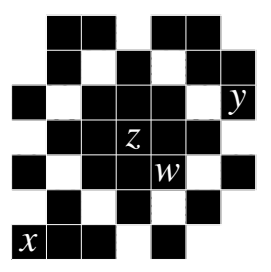

$X$

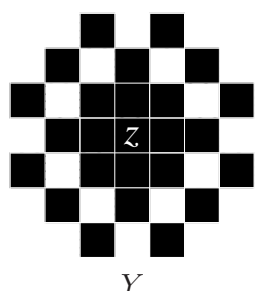

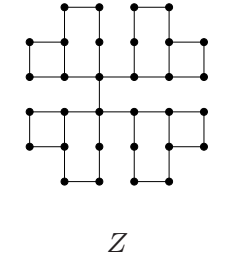

Fig. 12 Illustration of homotopic skeleton in $2 \mathrm{D}$ digital topology $(X, Y)$ and in cubical complexes $(Z)$ : the object $Y$ (black pixels) is an ultimate skeleton of $X$ (black pixels). The foreground object in black is equipped with the indirect adjacency relation (also called 8-adjacency relation) and its complement, the background object in white, is equipped with the direct adjacency relation (also called 4 -adjacency relation). A homotopic skeleton (namely an ultimate collapse) $Z$ of the two black objects $X$ and $Y$ considered in the framework of cubical complexes.

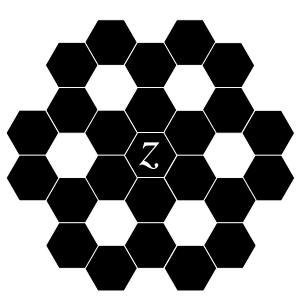

(a)

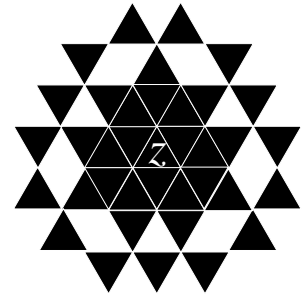

(b)
Fig. 13 (a) A homotopic skeleton in the hexagonal grid. The 6-adjacency relation is used for both the foreground (black) and background (white) objects. Any hexagon that is 6-adjacent to the black hexagon $z$ is also black. (b) A homotopic skeletion in the triangular grid. In this grid, we consider that any two black triangles that share a side or a vertex are adjacent. Note that any triangle adjacent to the black triangle $z$ is also black. Thus, the two objects in (a) and (b) may be considered as "thick objects".

in the sense that its dimension is always lower than the one of the pseudomanifold.

Let $X \preceq \mathbb{M}$, and let $y$ be any face of $X$. If there exists a unique face $x$ of $X$ which strictly includes $y$, we say that $y$ is a free face for $X$ and that the pair $(x, y)$ is a free pair for $X$. If the pair $(x, y)$ is a free pair for $X$ and if $d=\operatorname{dim}(x)$, then $(x, y)$ is called a free $d$-pair for $X$.

For instance, in Fig. 10b, $y$ is a free 1-face for the complex $X$ represented in black since $x$ is the only face of $X$ that strictly includes $y$. On the other hand, $z$ is not a free face since it is included in two distinct 2-faces of $X$. Therefore, $(x, y)$ is a free 2-pair for $X$ whereas $(x, z)$ is not. In Fig. $10 \mathrm{~d},\left(x^{\prime}, y^{\prime}\right)$ is a free 1 -pair for the complex represented in black.

Let $X$ be a subcomplex of $\mathbb{M}$ and let $(x, y)$ is a free $d$-pair for $X$. It can be seen that $x$ is necessarily a facet of $X$ (otherwise there would be a face $z$ that strictly includes $x$, and thus $y$ would be included in two faces of $X)$. Hence, the set $X \backslash\{x\}$ is a complex. Furthermore, the face $y$ is not strictly included in any face of $X \backslash\{x\}: y$ is a facet of $X \backslash\{x\}$. Thus, the set $(X \backslash\{x\}) \backslash\{y\}=X \backslash\{x, y\}$ is a complex.

Let $X \preceq \mathbb{M}$. If $(x, y)$ is a free pair for $X$, the complex $X \backslash\{x, y\}$ is an elementary collapse of $X$ or, more precisely, an elementary $d$-collapse of $X$ if $(x, y)$ is a free $d$-pair for $X$.

To illustrate this definition, let us consider the two complexes $X$ and $Y$ drawn in black in Figs. 10b and c respectively. Since $(x, y)$ is a free 2-pair for $X$ and since $Y=X \backslash\{x, y\}$, the complex $Y$ is an elementary 2-collapse of $X$.

Let $X$ and $Y$ be two subcomplexes of $\mathbb{M}$. The complex $Y$ is a collapse of $X$ if there exists a collapse sequence from $X$ to $Y$, i.e., a sequence of complexes $\left\langle X_{0}, \ldots, X_{\ell}\right\rangle$, with $\ell \geqslant 0$, such that $X_{0}=$ $X, X_{\ell}=Y$ and $X_{i}$ is an elementary collapse of $X_{i-1}$, for any $i$ in $\{1, \ldots, \ell\}$. If each $X_{i}$ is an elementary $d$ collapse of $X_{i-1}$, we also say that $Y$ is a $d$-collapse of $X$.

Let $X, Y, Z$ and $W$ be the four subcomplexes in black in Figs. 10b,c,d and e respectively. The complex $Z$ is a 2-collapse of both $X$ and $Y$, and $W$ is a collapse of $X, Y$ and $Z$ which is also a 1-collapse of $Z$. Moreover, it can be seen that $Z$ does not contain any free 2-pair but that it contains free 1-pairs whereas $W$ does not contain any free 2-pair nor free 1-pair.

In the following, let us write $X \prec \mathbb{M}$, if $X \preceq \mathbb{M}$ and $X \neq \mathbb{M}$ and let us recall that $n=\operatorname{dim}(\mathbb{M})$.

Intuitively, one may guess that a complex strictly included in $\mathbb{M}$, but of dimension $n$, has a border. The following property, whose complete proof is given in the remaining part of the section, establishes this fact.

Property 6 Let $X \prec \mathbb{M}$. If $\operatorname{dim}(X)=n$, then necessarily there exists a free $n$-pair for $X$.

Note that, by definition of a free $n$-pair, the converse is also true.

As a direct consequence of Property 6, any ultimate collapse (i.e., any collapse that does not contain any free pair) of any subcomplex of $\mathbb{M}$ is thin in the sense that its dimension is at most $n-1$. Note that if $X$ is not a subcomplex of an $n$-pseudomanifold, the previous property is, in general, not true. Note also that this property appears in [15] in the case where the space is the cubical complex $\mathbb{F}^{n}$, which is a discrete manifold whose faces are made of points in $\mathbb{Z}^{n}$.

Before proving Property 6, let us first state a remark.

Remark 7 If $A$ is a nonempty star of $\mathbb{M}$, then any face $x$ in $A$ is included in an n-face of $A$ (otherwise, since $\mathbb{M}$ is pure we would not have $\operatorname{star}(A)=A)$. 
Proof (of Property 6) Since $\operatorname{dim}(X)=n$, there exists, by definition, a facet $x$ of $X$ such that $\operatorname{dim}(x)=n$. As $X \neq \mathbb{M}$, we have $\bar{X} \neq \emptyset$. Thus, by Remark 7 , there exists an $n$-simplex $y$ in $\bar{X}$. Since $\mathbb{M}$ is an $n$ pseudomanifold, there exists a path $\left\langle x_{0}, \ldots, x_{\ell}\right\rangle$ from $x$ to $y$ in $\mathbb{M}$. Let $i$ be the lowest index in $\{1, \ldots, \ell\}$ such that $x_{i} \in \bar{X}$ and let $z$ be the $(n-1)$-simplex such that $z=x_{i-1} \cap x_{i}$. Since $X$ is a complex and since $x_{i-1}$ belongs to $X$, we deduce that $z$ belongs to $X$. Since $\mathbb{M}$ is an $n$-pseudomanifold, the faces $x_{i-1}$ and $x_{i}$ are the only two $n$-faces of $\mathbb{M}$ that contain $z$. As $x_{i} \notin X$, the face $x_{i-1}$ is the only face of $X$ that contains $z$. Thus, $\left(x_{i-1}, z\right)$ is a free $n$-pair for $X$.

\section{Cuts}

Segmentation is the task of delineating objects of interest. In many cases, the result of such process is a set of connected regions lying in a background which constitutes the separation between regions. Intuitively, a cut is a separation that cannot be reduced without connecting some regions. Our aim being to study segmentations in pseudomanifolds, we introduce the notion of cuts of a set of simplices. Then, we present two important results. First, we state (Property 13) that in an $n$-pseudomanifold the dimension of a nonempty cut is always $n-1$. Secondly, we give an equivalence result (Theorem 15) between cuts and some subsets of the ultimate collapses. This last property leads to an efficient method to compute cuts in pseudomanifolds.

\subsection{Extensions}

Given a set of "seed components", which "mark" the regions of interest of the space, many segmentation methods (among them, watershed algorithms) look for a set of connected components such that each connected component contains one seed and each seed is included in one of these components. The notion of an extension, presented hereafter in the case of simplicial complexes, provides a definition for the results produced by this kind of methods. This notion was first introduced by G. Bertrand [3] for studying watersheds in vertexweighted graph and was adapted in [24] to the case of edge-weighted graphs. In this section, we give a definition of extensions in pseudomanifolds, and we show (Property 11) that the collapse operation can be used to obtain extensions.

Definition 8 (extension) Let $A$ and $B$ be two nonempty stars in $\mathbb{M}$. We say that $B$ is an extension of $A$ if $A \sqsubseteq B$, and if each connected component of $B$ includes exactly one connected component of $A$. We also say that $B$ is an extension of $A$ if $A$ and $B$ are both empty.

For instance, the sets of gray simplices in Figs. 10c-f are extensions of the set of gray simplices in Fig. 10b. Observe, in particular, that all these five sets of simplices include three connected components.

Remark 9 Let $A, B$ and $C$ be three stars in $\mathbb{M}$. If $B$ is an extension of $A$ and if $C$ is an extension of $B$, then clearly $C$ is an extension of $A$.

In fact, we have a more remarkable "triangular" property. This property, which is given below, can be derived from Theorem 4 in [3] stated in the framework of graphs. To this end, one may associate to $\mathbb{M}$ the graph whose vertices are the $n$-faces of $\mathbb{M}$ and whose edge-set is made of the pairs of $n$-faces whose intersection is an $(n-1)$-face of $\mathbb{M}$.

Property 10 (confluence, from [3]) Let $A, B$, and $C$ be three stars of $\mathbb{M}$ such that $C$ is an extension of $A$ and $A \sqsubseteq B \sqsubseteq C$. The star $B$ is an extension of $A$ if and only if $C$ is an extension of $B$.

The collapse operation preserves the topology of the object. The next result (Property 11) shows that it also preserves the number of connected components of the complement of the object when the object is a subset of an $n$-pseudomanifold (the property would not hold true without this hypothesis).

Property 11 Let $X \preceq \mathbb{M}$ and $Y$ be a collapse of $X$. Then $\bar{Y}$ is an extension of $\bar{X}$.

Proof We are going to establish the result in the case where $Y$ is an elementary collapse of $X$. Then, due to Remark 9, this will prove Property 11 by induction.

Let us suppose that $Y$ is an elementary collapse of $X$. Let $(x, y)$ be the free pair for $X$ such that $Y=$ $X \backslash\{x, y\}$. By construction $\bar{X} \sqsubseteq \bar{Y}$. Thus, to establish that $\bar{Y}$ is an extension of $\bar{X}$, we will prove that any component of $\bar{Y}$ includes exactly one component of $\bar{X}$. Let $C^{\prime}$ be any component of $\bar{Y}$, and let $C=C^{\prime} \backslash\{x, y\}$. Thus, we have $C^{\prime} \cap \bar{X}=C$. It can be seen that to establish the result, it is sufficient to show that (1) $C \neq \emptyset$ (hence that $C^{\prime}$ includes at least one connected component of $\bar{X}$ ) and that (2) $C$ is connected.

1) By definition $C^{\prime}$ is a nonempty star. Thus, $C^{\prime}$ contains at least one $n$-face $w$. If $w \neq x$, then $C \neq \emptyset$ since $w \in C$. If $w=x$, then $y \subseteq w$ is an $(n-1)$-face. Since $\mathbb{M}$ is an $n$-pseudomanifold, there exists an $n$ face $w_{1} \neq w$ such that $y \subseteq w_{1}$. Since $(x, y)$ is a free pair for $X$, the face $w_{1}$ belongs to $\bar{X}$. Since $\bar{X} \sqsubseteq \bar{Y}$, we have $w_{1} \in \bar{Y}$. It can be seen that the faces $w$ 
and $w_{1}$ are linked in $\bar{Y}$. Thus, we have $w_{1} \in C^{\prime}$ as $w$ and $w_{1}$ are linked in $\bar{Y}$. Thus, the $n$-face $w_{1}$ belongs to $C$, and $C \neq \emptyset$.

2) We are going to prove that any two $n$-faces $w$ and $z$ in $C$ are linked for $C$. Since $C \sqsubseteq C^{\prime}$ and $C^{\prime}$ is connected, there exists, from $w$ to $z$, a simple path $\pi=\left\langle w_{0}, \ldots, w_{\ell}\right\rangle$ in $C^{\prime}$.

2.i) Let us first prove that, for any $i \in\{0, \ldots, \ell\}$, we have $w_{i} \in C$. As $w_{0}=w$ and $w_{\ell}=z$, the faces $w_{0}$ and $w_{\ell}$ belong to $C$. Let $i \in\{1, \ldots, \ell-1\}$. The two $(n-1)$-faces $w_{i-1} \cap w_{i}$ and $w_{i} \cap w_{i+1}$ belong to $C^{\prime}$. Since $\mathbb{M}$ is a $n$-pseudomanifold and $\pi$ a simple path, we have $w_{i-1} \cap w_{i} \neq w_{i} \cap w_{i+1}$ (otherwise the $(n-1)$-face $w_{i-1} \cap w_{i}$ would be included in three distinct $n$-faces). Hence, by definition of $C$, at least one of $w_{i-1} \cap w_{i}$ and $w_{i} \cap w_{i+1}$ belongs to $C$. Thus, since $C$ is a star, the face $w_{i}$ belongs to $C$.

2.ii) Suppose now that $\pi$ is not a path in $C$. Thus, there exists $i \in\{1, \ldots, \ell\}$ such that either $w_{i} \cap w_{i-1}=y$ or $w_{i} \cap w_{i-1}=x$. Suppose first that $w_{i} \cap w_{i-1}=y$. Thus $y$ is an $(n-1)$-face. Hence, $x$ is an $n$-face. Since $\pi$ is a simple path, we have $w_{i} \neq w_{i-1}$. Thus, since $\mathbb{M}$ is a $n$-pseudomanifold, we have $x=w_{i}$ or $x=w_{i-1}$. Thus, one of $w_{i-1}$ and $w_{i}$ belongs to $X$, that is a contradiction with (2.i). Thus, necessarily, we have $w_{i} \cap w_{i-1}=x$. Therefore, $y$ is an $(n-2)$ face included in both $w_{i}$ and $w_{i-1}$. Furthermore, the face $y$ belongs to $\bar{Y}$. By Lemma 5 , the $n$ faces $w_{i-1}$ and $w_{i}$ are linked for $\operatorname{star}(y) \backslash\{x\}$ by a path $\pi^{\prime}=\left\langle z_{0}, \ldots, z_{m}\right\rangle$ in $\operatorname{star}(y) \backslash\{x\}$ such that $z_{0}=w_{i-1}$ and $z_{m}=w_{i}$. Since $\bar{Y}$ is a star and since $y \in \bar{Y}$, we have $\operatorname{star}(y) \sqsubseteq \bar{Y}$. Hence, $\pi^{\prime}$ is a path in $\bar{Y}$. Furthermore, since $C^{\prime}$ is the connected component of $\bar{Y}$ that contains $w_{i-1}$ and $w_{i}$, we deduce that $\pi^{\prime}$ is a path in $C^{\prime}$. Thus, the path $\pi^{\prime \prime}=\left\langle w_{0}, \ldots, w_{i-1}=z_{0}, \ldots, z_{m}=w_{i}, \ldots, w_{\ell}\right\rangle$ is a path in $C^{\prime}$. By construction, we have $w_{j} \cap w_{j-1} \neq x$, for any $j \in\{1, \ldots, \ell\}$ and $z_{j} \cap z_{j-1} \neq x$, for any $j \in\{1, \ldots, m\}$. As $C=C^{\prime} \backslash\{x, y\}, \pi^{\prime \prime}$ is also a path in $C$, that is $w=w_{0}$ and $z=w_{\ell}$ are linked for $C$.

Let us first illustrate Property 11 with the subcomplexes $X, Y, Z$, and $W$, drawn in black in Figs. 10b-e respectively. The subcomplexes $Y, Z$, and $W$ are three collapses of $X$, and therefore $\bar{Y}, \bar{Z}$ and $\bar{W}$ are three extensions of $\bar{X}$. In particular, these four stars all include exactly three components.

Let us also illustrate the previous property in the pinched 2-pseudomanifold of Fig. 9b. Let $X$ and $Y$ be the two subcomplexes represented in black in Figs. 14a and b, respectively. The complementary set $\bar{X}$ of $X$ includes two components. Let $x$ be a triangle located at the left of the pinching, let $y$ be a triangle located at the right of the pinching, and let $z=x \cap y$ be the pinching. Observe that there is no path in $\bar{X}$ from $x$ to $y$. Thus, one of the two components of $\bar{X}$ contains the face $x$ and the the second one contains the face $y$. The subcomplex $Y$ is a collapse of $X$. Therefore, by Property 11, the star $\bar{Y}$ is an extension of $\bar{X}$. Thus, as $\bar{X}$, the star $\bar{Y}$ includes two components. Indeed, even if the point $z$ belongs to $\bar{Y}$, there is no path from $x$ to $y$ in $\bar{Y}$. In particular, the sequence $\langle x, y\rangle$ is not a path, according to the definition of $d$-path given in Section 2 (here $d=2$ ), since $\operatorname{dim}(z) \neq 1$. Note also that the point $z$ does not belong to any component of $\bar{Y}$ since there is no connected substar of $\bar{Y}$ that contains $z$. In fact, the definition of connected component adopted in this paper falls into the category of partial connections, which are investigated by $\mathrm{C}$. Ronse in [50], and which do not require that any element of a set belongs to a connected component of this set. This second example also illustrates our choice of considering the notion of $d$-path (with $d=n$ ) for the connectivity of the complement of the simplicial complexes (i.e. the star connectivity).

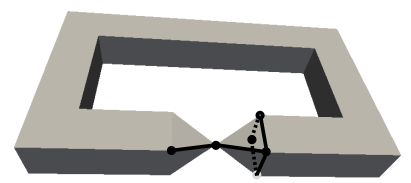

(a) $X$ (in black)

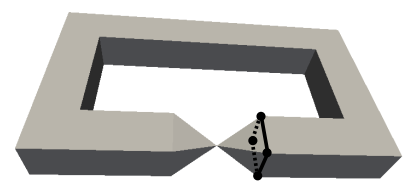

(b) $Y$ (in black)
Fig. 14 Illustration of Property 11 where the subcomplex $Y$ is a collapse of $X$.

\subsection{Cuts}

Among the segmentation methods that produce extensions, several aim at building maximal extensions, i.e. extensions of the seeds that are not included in any strictly larger extension of the seeds. In this case, the set that separates the connected components of the extension is called a cut. The notion of a cut corresponds to the intuitive idea of a frontier between regions. We prove in this section that the dimension of a nonempty cut is always $n-1$ in an $n$-pseudomanifold.

Definition 12 (Cut) Let $A$ be a nonempty star in $\mathbb{M}$ and $X$ be a subcomplex of $\mathbb{M}$. We say that $X$ is a cut for $A$ if $\bar{X}$ is an extension of $A$ and if $X$ is minimal for this property, i.e., for any $Y \preceq X$, if $\bar{Y}$ is an extension of $A$, then we have necessarily $Y=X$.

Observe that there can be several distinct cuts for a same star $A$ and, in this case, these distinct cuts 
do not necessarily contain the same number of faces. Thus, a cut for a star $A$ is not necessarily a smallest complex in the number of faces among the complexes whose complement is an extension of $A$. However, as we will see with the next property, a cut is always "thin". The set of black simplices in Fig. 10f is a cut for $\bar{X}$ (where $X$ is the complex depicted in black in Fig. 10b).

Intuitively, a "frontier" or a cut in an $n$-dimensional space should be an object of dimension $n-1$. Nevertheless, we have shown [21] that the clefts (a notion in the framework of digital topology that corresponds to cuts in the framework of this paper) in the grids $\mathbb{Z}^{2}, \mathbb{Z}^{3}, \mathbb{Z}^{n}$ equipped with usual adjacency relations [31] cannot be considered as $(n-1)$-dimensional objects (the clefts are not necessarily thin). For instance, the set $Y$ of black pixels in Fig. 12 is a cleft, however, we have seen in Section 3 that this set is not thin. From this point of view, the next result shows that the framework of pseudomanifolds is an interesting alternative to usual adjacency relations, as used in digital topology.

Property 13 Let $A \sqsubseteq \mathbb{M}$ and $X \preceq \mathbb{M}$, with $A \neq \emptyset$. If $X$ is a cut for $A$, then the complex $X$ is either empty or a pure $(n-1)$-complex.

Proof We first prove by contradiction that $\operatorname{dim}(X)<$ $n$. Suppose that $\operatorname{dim}(X)=n$. Then, by Property 6 , there exists a free $n$-pair for $X$. Hence, there exists an $n$ collapse $Y$ of $X$ such that $Y \prec X$. By Property $11, \bar{Y}$ is an extension of $\bar{X}$, which is itself an extension of $A$ (Definition 12). Thus, by Remark $9, \bar{Y}$ is also an extension of $A$, a contradiction with the fact that $X$ is a cut and $Y \prec X$. Thus, $\operatorname{dim}(X)<n$.

Suppose that $X$ is nonempty. We will now prove that $X$ is pure. To this end, we have to prove that the dimension of any facet $x$ of $X$ equals $n-1$. Let $Y=$ $X \backslash\{x\}$. Since $x$ is a facet of $X, Y$ is a complex. Since $X$ is a cut, $\bar{Y}$ is not an extension of $A$ (Definition 12). From the definition of $X$ and $Y$, it can be seen that any component of $A$ is included in a component of $\bar{Y}$. Thus, there exists a component $B$ of $\bar{Y}$ which includes at least two nonempty components $A_{1} \sqsubseteq A$ and $A_{2} \sqsubseteq A$ of $A$. Since $A_{1}$ and $A_{2}$ are nonempty stars, there exist two $n$ simplices $y$ and $z$ such that $y \in A_{1}$ and $z \in A_{2}$. Since $B$ is connected, there exists a path $\pi=\left\langle x_{0}, \ldots, x_{\ell}\right\rangle$ in $B$ such that $x_{0}=y$ and $x_{\ell}=z$. Since $\bar{X}$ is an extension of $A, \pi$ is not a path in $\bar{X}$. But, for any $i$ in $\{0, \ldots, \ell\}$, we have $\operatorname{dim}\left(x_{i}\right)=n$, and, therefore, the face $x_{i}$ is in $\bar{X}$, since $\operatorname{dim}(X)<n$. Thus, there exists an index $i \in$ $\{1, \ldots, \ell\}$ such that the $(n-1)$-face $x_{i-1} \cap x_{i}$ is in $X$. On the contrary, by definition of $\pi$, the face $x_{i-1} \cap x_{i}$ is in $\bar{Y}$. Thus, by construction of $Y$, we have $x_{i-1} \cap x_{i}=x$. Hence, we have $\operatorname{dim}(x)=n-1$.
We remark that Definition 12 does not directly lead to an efficient algorithm to compute a cut. Indeed, based on this definition, in order to obtain a cut for a star $A$, one needs i) to consider all the complexes included in $\bar{A}$; ii) to remove from this family the complexes whose complement is not an extension of $A$ (this requires to find the inclusion relationship between components of the complement of the considered complexes and those of $A$ ); and iii) to find among the remaining complexes one element that is minimal for the inclusion relation. Step i) alone has an exponential complexity.

\subsection{Cut by collapse}

In this section, we introduce the notion of a cut by collapse. Intuitively, a cut by collapse for a star $A$, which correspond e.g. to the seeds in a segmentation task, is a complex made of all faces of an ultimate collapse of $\bar{A}$ that are adjacent to at least two components of the complement of this collapse. The main result of this section is an equivalence theorem (Theorem 15) between the cuts for $A$ and the cuts by collapse for $A$. A simple method is presented to compute cuts by collapse, hence cuts, in linear-time.

We introduce hereafter the notion of multiconnected faces that will play a fundamental role for cuts by collapse.

Let $X \preceq \mathbb{M}$, let $A \sqsubseteq \mathbb{M}$ and $x$ be an $(n-1)$-face of $\mathbb{M}$. We say that $x$ is adjacent to $A$ if there exists a simplex $y \in A$ such that either $x \subseteq y$ or $y \subseteq x$. An $(n-1)$-face of $X$ is said to be multi-connected for $X$ if it is adjacent to at least two components of $\bar{X}$.

For instance, the set of faces that are multiconnected for the complex in black in Fig. 10d is composed of the 1-faces represented in black in Fig. $10 \mathrm{f}$. We remind that the simplicial closure $\operatorname{close}(X)$ of a set of faces $X$ is the complex made of all subsets of the elements in $X$.

Definition 14 (cut by collapse) Let $A$ be a substar of $\mathbb{M}$, and let $X$ be a subcomplex of $\mathbb{M}$.

We say that the complex $X$ is a cut by collapse for $A$ if there exists a complex $Y \preceq \mathbb{M}$ such that:

1. $Y$ is a collapse of $\bar{A}$;

2. $Y$ does not contain any free $n$-pair; and

3. $X$ is the simplicial closure of the set of $(n-1)$-faces in $Y$ that are multiconnected for $Y$.

For instance, the complex $V$ in black in Fig. 10f is a cut by collapse for the star $A=\bar{X}$ represented in gray in Fig. 10b. Indeed, the complex $Z$ (black faces of Fig. 10d) is a 2-collapse of $X$ that does not contain any 
free 2-pair and $V$ is the closure of the set of all faces that are multi-connected for $Z$.

To obtain a cut by collapse $Y$ for an object $A$, the following straightforward algorithm can be used:

1. While $\bar{A}$ contains a free $n$-pair do

- Select a free $n$-pair $(x, y)$ for $\bar{A}$;

- Add $x$ and $y$ to $A$ (i.e. $A:=A \cup\{x, y\}$ )

2. Set $Z$ to the set of all multi-connected faces for $A$.

3. Set $Y$ to the simplicial closure of $Z$.

Each step of elementary collapse requires only a local test. Moreover, the use of a breadth-first strategy ([17], Chapter 22) leads to a linear-time algorithm for performing step 1. To perform Step 2, one stage of global computation (connected component labeling) is required. Therefore step 2 can also be performed in linear-time. Furthermore, Step 3 can be performed on the fly while doing step 2. Thus, the overall complexity of the previous algorithm is linear. Observe also that, according to the definition of a cut by collapse, if we replace the free $n$-pairs by free pairs in the preceding algorithm, then the algorithm would still output a cut by collapse. Nevertheless, this algorithm would require more iterations of elementary collapse to achieve the same result.

The following result establishes that any complex which is a cut is a cut by collapse, and that the converse is also true. Therefore, the above algorithm constitutes a simple and linear-time algorithm to compute cuts.

Theorem 15 Let $A \sqsubseteq \mathbb{M}$. A complex $X$ is a cut for $A$ if and only if it is a cut by collapse for $A$.

The remaining part of the section is devoted to the proof of Theorem 15. To this end, we will first prove the forward implication of the theorem (Property 17) and then the backward implication (Property 19).

Lemma 16 Let $X \preceq \mathbb{M}$ such that $\operatorname{dim}(X)<n$. Let $Y$ be the closure of the set of all faces of $X$ that are multiconnected for $X$.

\section{If $X \backslash Y \neq \emptyset$, then $X \backslash Y$ contains a facet of $X$.}

Let $x$ be a facet of $X$ such that $x$ belongs to $X \backslash Y$ and let $Z=X \backslash\{x\}$.

2. The subset $Z$ of $\mathbb{M}$ is a complex.

3. The star $\bar{Z}$ is an extension of $\bar{X}$.

4. The complex $Y$ is exactly the closure of the set of all faces of $Z$ that are multiconnected for $Z$.

Proof 1) Since $X \backslash Y \neq \emptyset$, there exists $y \in X \backslash Y$. If $y$ is a facet of $X$, the proof is done. If $y$ is not a facet of $X$, then necessarily there exists a facet $z$ of $X$ that includes $y$. As $Y$ is the closure of the set of all faces of $X$ that are multiconnected for $X$ and as $y \notin Y$, we have $z \notin Y$.

2) follows directly from the fact that $x$ is a facet of $X$.

3 ) will be proved by contradiction. To this end, let us suppose that $\bar{Z}$ is not an extension of $\bar{X}$. By construction, $\bar{X} \sqsubseteq \bar{Z}$. Thus, any component of $\bar{X}$ is included in one component of $\bar{Z}$. Since, by hypothesis, $\bar{Z}$ is not an extension of $\bar{X}$, there must exist one component $A$ of $\bar{Z}$ that does not include any component of $\bar{Z}$ or that includes at least two distinct nonempty components $B$ and $C$ of $\bar{X}$. In the former case, since $\bar{Z}=\bar{X} \cup\{x\}$, we deduce that $A=\{x\}$, which is impossible since $\operatorname{dim}(x)<n$ and $A$ is a star. Let us now consider the latter case. In this case, since $B$ and $C$ are nonempty stars, there exists a simple path $\pi=\left\langle x_{0}, \ldots, x_{\ell}\right\rangle$ in $\bar{Z}$ such that $x_{0}$ is an $n$-simplex of $B$ and $x_{\ell}$ is an $n$-simplex of $C$. As $\pi$ is a path in $\bar{Z}$, for any $i \in\{1, \ldots, \ell\}$, the face $x_{i-1} \cap x_{i}$ is an $(n-1)$-simplex of $\bar{Z}$. Since $x_{0}$ and $x_{\ell}$ belong to distinct components of $\bar{X}, \pi$ is not a path in $\bar{X}$. As $\operatorname{dim}(X)<n$, for any $i \in\{0, \ldots, \ell\}$, we have $x_{i} \in \bar{X}$. Thus, there exists $i \in\{1 \ldots \ell\}$ such that $x_{i-1} \cap x_{i}$ does not belong to $\bar{X}$. As $Z=X \backslash\{x\}$, we have $x_{i-1} \cap x_{i}=x$. From the preceding remarks, we deduce that $\left\langle x_{0}, \ldots, x_{i-1}\right\rangle$ and $\left\langle x_{i}, \ldots, x_{\ell}\right\rangle$ are two paths in $\bar{X}$. Since $x_{0}$ and $x_{\ell}$ belong to distinct components of $\bar{X}$ and since $x$ is a $(n-1)$-face of $X$ such that $x \subseteq x_{i-1}$ and $x \subseteq$ $x_{i}$, we deduce that $x$ is multiconnected for $X$, which constitutes a contradiction with $x \in X \backslash Y$.

4) Let $y$ be an $(n-1)$-face of $Z$. To establish 4$)$, we are going to prove that $y$ is multiconnected for $Z$ if and only if $y$ is multiconnected for $X$. Since $\mathbb{M}$ is an $n$-pseudomanifold, there exists exactly two distinct $n$-faces $z_{1}$ and $z_{2}$ of $\mathbb{M}$ that contain $y$. These two $n$ faces belong to both $\bar{X}$ and $\bar{Z}$ (since $\operatorname{dim}(X)<n$ and since $Z=X \backslash\{x\})$. Since $\bar{Z}$ is an extension of $\bar{X}$, the $n$-faces $z_{1}$ and $z_{2}$ belong to the same component of $\bar{Z}$ if and only if they belong to the same component of $\bar{X}$. Thus $y$ is multiconnected for $Z$ if and only if $y$ is multiconnected for $X$.

Property 17 Let $A \sqsubseteq \mathbb{M}$. If $X$ is a cut by collapse for $A$, then $X$ is a cut for $A$.

Proof By definition of a cut by collapse, there exists a collapse $Y$ of $\bar{A}$ such that $Y$ does not contain any free $n$-pair and such that $X$ is the closure of the set of all $(n-1)$-faces of $Y$ that are multiconnected for $Y$. By Property $11, \bar{Y}$ is an extension of $A$. We will now establish that $\bar{X}$ is an extension of $\bar{Y}$, which, by Remark 9, will prove that $\bar{X}$ is an extension of $A$. Let us consider a sequence $\left\langle Y_{0}, \ldots, Y_{\ell}\right\rangle$ of subcomplexes of $\mathbb{M}$ such that: 
1. $Y_{0}=Y$; and

2. $Y_{i}=Y_{i-1} \backslash\left\{x_{i-1}\right\}$, where $x_{i-1}$ is a facet of $Y_{i-1}$ that is not included in a face that is multiconnected for $Y_{i-1}$; and

3. any facet of $Y_{\ell}$ is multiconnected for $Y_{\ell}$.

Since $Y$ does not contain any free $n$-pair, by the contraposition of Property 6 , we have $\operatorname{dim}(Y)<n$. Hence, by Lemma 16.3, the star $\overline{Y_{i}}$ is an extension of $\overline{Y_{i-1}}$. Therefore, due to Remark 9 , the star $\overline{Y_{i}}$ is, by induction, an extension of $\overline{Y_{0}}=\bar{Y}$. Due to Lemma 16.4, we also deduce, by induction, that $X$ is the closure of the set of faces of $Y_{i}$ that are multiconnected for $Y_{i}$. By the contraposition of Lemma 16.1, we have $Y_{\ell}=X$. Thus, the star $\bar{X}$ is an extension of $A$.

In order to establish that $X$ is a cut for $A$, it remains to show that, for any $Z \preceq X$, if $\bar{Z}$ is an extension of $A$, then we have necessarily $Z=X$. Let $Z \preceq X$ such that $\bar{Z}$ is an extension of $A$. Suppose that $Z \neq X$. Then, there exists $x \in X \backslash Z$. Since $X=Y_{\ell}$, the dimension of any facet of $X$ is not greater than $n-1$ and any facet of $X$ is multiconnected. Thus, there exists an $(n-1)$-face $y \in X$ such that $x \subseteq y$ and such that $y$ is multiconnected for $X$. Thus, there exist two $n$ faces $z, w \in \bar{X}$ such that, $y \subseteq z, y \subseteq w$, and $z$ and $w$ belong to two distinct components of $\bar{X}$. Since $Z$ is a complex, $x \notin Z$ and $x \subseteq y$ imply $y \notin Z$. Thus, $\langle z, w\rangle$ is a path in $\bar{Z}$. Hence, the connected component of $\bar{Z}$ that contains $y$ includes at least two components of $\bar{X}$, and, since $\bar{X}$ is an extension of $A$, this component of $\bar{Z}$ includes also at least two components of $A$. Thus, the star $\bar{Z}$ is not an extension of $A$, a contradiction.

Lemma 18 Let $A \sqsubseteq \mathbb{M}$ and let $X$ be a cut for $A$. If $\operatorname{dim}(\bar{A})=n$, then there exists a free $n$-pair $(z, w)$ for $\bar{A}$ such that $z$ and $w$ are in $\bar{X}$.

Proof If $X=\emptyset$, the, Property 6 is sufficient to establish Lemma 18. From now on, suppose that $X \neq$ $\emptyset$. Since $\operatorname{dim}(\bar{A})=n$, there exists $x \in \bar{A}$ such that $\operatorname{dim}(x)=n$. By Property 13, we have $\operatorname{dim}(X)=$ $n-1$. Thus $x$ is in $\bar{X}$. Let $B$ be the connected component of $\bar{X}$ which contains $x$. Since $X$ is a cut for $A$, there exists a nonempty component $B^{\prime}$ of $A$ such that $B^{\prime} \sqsubseteq B$. Since $B^{\prime}$ is a star, there exists $y \in B^{\prime}$ whose dimension is $n$. Since both $x$ and $y$ belong to $B$ and since $B$ is a connected star, there exists a path $\pi=\left\langle x_{0}, \ldots, x_{\ell}\right\rangle$ in $B$ such that $x_{0}=x$ and $x_{\ell}=y$. Since $x \in \bar{A}$ and $B^{\prime}$ is a component of $A$, the face $x$ is not in $B^{\prime}$. Since $y \in B^{\prime}$, there exists an index $i \in$ $\{1, \ldots, \ell\}$ such that $x_{i} \in B^{\prime}$ whereas $x_{i-1} \notin B^{\prime}$. By definition of a path, $x_{i} \cap x_{i-1}=w$ is a $(n-1)$ face. Since $x_{i-1}$ does not belong to $B^{\prime}$, which is a connected component of $A$, we have $x_{i-1} \in \bar{A}$. As $\bar{A}$ is a complex and as $w \subseteq x_{i-1}, w \in \bar{A}$. By definition of $B^{\prime}$, the face $x_{i}$ is in $A$. Thus, since $\mathbb{M}$ is an $n$ pseudomanifold, we deduce that $x_{i-1}$ is the only $n$-face of $\bar{A}$ which contains $w$. Thus, $\left(x_{i-1}, w\right)$ is a free $n$-pair for $\bar{A}$. Furthermore, we remind that $\pi$ is a path in $B$. Thus, $x_{i-1} \in B$ and $x \in B$. But $B \subseteq \bar{X}$, thus $x_{i-1}$ and $w$ are in $\bar{X}$.

Property 19 Let $A \sqsubseteq \mathbb{M}$ and let $X$ be a cut for $A$. Then, $X$ is a cut by collapse for $A$.

Proof Let us consider a sequence $\left\langle X_{0}, \ldots, X_{\ell}\right\rangle$ of complexes such that:

1. $X_{0}=\bar{A}$;

2. for any $i \in\{1, \ldots, \ell\}, X_{i}=X_{i-1} \backslash\{x, y\}$ where $(x, y)$ is a free $n$-pair for $X_{i-1}$ such that $x \notin X$ and $y \notin X$; and

3. Any free $n$-pair $(x, y)$ for $X_{\ell}$ is such that $x \in X$ or $y \in X$.

Clearly, $X_{\ell}$ is an $n$-collapse of $X_{0}=\bar{A}$. By the contraposition of Lemma 18, $\operatorname{dim}\left(X_{\ell}\right)<n$. Thus, $X_{\ell}$ does not contain any free $n$-pair. Hence, the closure $S$ of the set of all $(n-1)$-faces which are multiconnected for $X_{\ell}$ is a cut by collapse for $A$. Therefore, by Property $17, S$ is a cut for $A$. To complete the proof of Property 19, we will establish that $X \subseteq S$. Indeed, since $X$ and $S$ are cuts for $A$, this will prove, by the minimality property of cuts, that $S=X$, hence that $X$ is a cut by collapse for $A$.

Let $x \in X$ and $x^{\prime}$ be a facet of $X$ that contains $x$. Since $X$ is a cut for $A$, by Property $13, \operatorname{dim}\left(x^{\prime}\right)=n-1$. Since $X \subseteq \bar{A}$ and $\bar{A}=X_{0}$, we have $X \subseteq X_{0}$. Thus, since, for any $i$ in $\{1, \ldots, \ell\}$, we have $\left(X_{i-1} \cap X\right) \subseteq X_{i}$, we deduce that $X \subseteq X_{\ell}$. Hence, we have $x^{\prime} \in X_{\ell}$ and thus also $x \in X_{\ell}$ since $X_{\ell}$ is a complex and $x \subseteq x^{\prime}$. As $\mathbb{M}$ is an $n$-pseudomanifold, there exist two distinct $n$-faces $x_{0}$ and $y_{0}$ of $\mathbb{M}$ that contains $x^{\prime}$. Since $\operatorname{dim}\left(X_{\ell}\right)<n, x_{0}$ and $y_{0}$ belong to $\overline{X_{\ell}}$. As $X_{\ell}$ is an $n$-collapse of $\bar{A}$, by Property $11, \overline{X_{\ell}}$ is an extension of $A$. Thus, there are two paths $\pi_{x}=\left\langle x_{0}, \ldots, x_{k}\right\rangle$ and $\pi_{y}=\left\langle y_{0}, \ldots, y_{m}\right\rangle$ in $\overline{X_{\ell}}$ such that $x_{k}$ and $y_{m}$ belong to $A$. Since $\overline{X_{\ell}} \subseteq \bar{X}$ (see above), $\pi_{x}$ and $\pi_{y}$ are paths in $\bar{X}$. Since $X$ is a cut, $x_{k}$ and $y_{m}$ belong to distinct components of $A$ (otherwise we would have $X \backslash\left\{x^{\prime}\right\} \preceq X$ and $\overline{X \backslash\left\{x^{\prime}\right\}}$ would be an extension of $A$, a contradiction with the fact that $X$ is a cut). Thus, $x^{\prime}$ is multiconnected for $X_{\ell}$. Hence, $x \in S$. $\square$

\section{Simplicial stacks}

This section presents some basic definitions relative to maps defined on a pseudomanifold. In particular, we introduce the simplicial stacks as the maps whose level 
sets are all simplicial complexes. This notion will be used in the next section to easily extend the operation of collapse from complexes to maps.

Here and subsequently $k_{\min }$ and $k_{\max }$ stand for two elements of $\mathbb{Z}$ such that $k_{\text {min }}<k_{\text {max }}$. We set $\mathbb{K}=\{k \in$ $\left.\mathbb{Z} \mid k_{\min } \leqslant k \leqslant k_{\max }\right\}$.

Let $F$ be any map from $\mathbb{M}$ into $\mathbb{K}$. For any face $x$ of $\mathbb{M}$, the value $F(x)$ is called the altitude of $x$ (for $F$ ). Let $k \in \mathbb{K}$. The $k$-section of $F$, denoted by $F[k]$, is the set of faces of $\mathbb{M}$ whose altitude is greater than or equal to $k: F[k]=\{x \in \mathbb{M} \mid F(x) \geqslant k\}$.

Definition $20 A$ (simplicial) stack $F$ (on $\mathbb{M}$ ) is a map from $\mathbb{M}$ into $\mathbb{K}$ such that, for any $k \in \mathbb{K}$, the $k$-section of $F$ is a simplicial complex.

A set $X$ being a complex whenever it is equal to its closure (i.e., whenever we have $X=\operatorname{close}(X)$ ), we can see that a map $F$ is a simplicial stack if any $k$ section $F[k]$ of $F$ is equal to its closure (i.e., $F[k]=$ close $(F[k]))$. By duality (see Property 3 ), we can also see that $F$ is a simplicial stack if and only if, for any $k \in \mathbb{K}$, the set of faces of $\mathbb{M}$ whose altitude is less than $k$ is a star. In other words, a map $F$ is a simplicial stack if, for any two faces $x$ and $y$ of $\mathbb{M}$ such that $x \subseteq y$, we have $F(x) \geqslant F(y)$. Fig. 15 a depicts a stack $F$ and Figs. 15b, c and d depict in black the $k$-sections of $F$ for respectively $k=1,2,3$. These $k$-sections are indeed simplicial complexes.

Remark 21 Observe that we can obtain a stack $H$ from any map $F$ by considering the simplicial closure of $F$, i.e., $H$ is the simplicial stack such that, for any $k \in \mathbb{K}$, the $k$-section of $H$ is the simplicial closure of the $k$-section of $F$. Note also that the simplicial closure $H$ of a map $F$ can be easily obtained by setting $H(x)$ to the maximum altitude for $F$ of the simplices which include $x$ (i.e. $H(x)=\max \{F(y) \mid x \subseteq$ $y$ and $y \in \mathbb{M}\}$ ), for any face $x$ of $\mathbb{M}$.

Important notation 22 In the sequel of this paper, the symbol $F$ denotes a simplicial stack on $\mathbb{M}$.

We finish this section by presenting the notion of a (regional) minimum of a stack, which plays an important role for defining a watershed. By the very definition of a stack, the set of faces whose altitude is less than a given value $k$ is a star. Thus, the minima of a stack are also necessarily stars.

Let $A \sqsubseteq \mathbb{M}$ and let $k \in \mathbb{K}$. We say that $A$ is a minimum of $F$ (at altitude $k$ ) if $A$ is a connected component of $\overline{F[k+1]}$ and if $A \cap \overline{F[k]}=\emptyset$. In the following, we denote by $\mathcal{M}_{F}$ the union of all minima of $F$.

In other words, a connected substar $A$ of $\mathbb{M}$ is a minimum of $F$ at altitude $k$ if the altitude of any face in $A$ is $k$ and if the altitude of any face in the boundary $\operatorname{close}(A) \backslash A$ of $A$ (i.e., any face of $\bar{A}$ included in a face of $A$ ) is strictly greater than $k$.

Observe that, by definition, any minimum of $F$ is a star and therefore $\mathcal{M}_{F}$ is also a star.

For instance, the star made of all faces that belong to the minima of the stack $F$ represented in Fig. 15a are depicted in gray in Fig. 15e.

\section{Collapses of simplicial stacks}

We propose an operation of a collapse of a simplicial stack based on the collapse operation in the sections of the stack. In this framework, an ultimate collapse of a stack can be seen as an analog of a homotopic grayscale skeleton in digital topology $[7,45]$.

Let $y$ be any face of $\mathbb{M}, d-1$ be the dimension of $y$ and $k=F(y)$. If $y$ is a free face for $F[k]$, we say that $y$ is a free face for $F$. If $y$ is a free face for $F$, there exists a unique face $x$ in $F[k]$ such that $(x, y)$ is a free pair for $F[k]$ and we say that the pair $(x, y)$ is a free pair or a free $d$-pair for $F$. Let $(x, y)$ be a free pair for $F$. Then, $(x, y)$ is also a free pair for $F[k]$. Thus, $x$ is a face of $F[k]$, and we have $y \subseteq x$. Therefore, we have $F(x) \geqslant k$ and $F(x) \leq F(y)$ (since $F$ is a stack) which imply that $F(x)=F(y)=k$.

In Fig. 15a, the 1 -face $y$ at altitude 1 is a free face for the depicted map $F$. Indeed, $y$ is a free face for $F[1]$ (Fig. 15b). Thus, the pair $(x, y)$ in Fig. 15a is a free pair for $F$.

Let $S \subseteq \mathbb{M}$. The indicator function of $S$, denoted by $\mathbb{1}_{S}$, is the map from $\mathbb{M}$ into $\mathbb{K}$ such that $\mathbb{1}_{S}(x)=1$ for any $x$ in $S$ and such that $\mathbb{1}_{S}(x)=0$ for any $x$ in $\bar{S}$. The lowering of $F$ at $S$ is the map $F-\mathbb{1}_{S}$ from $\mathbb{M}$ into $\mathbb{K}$. Thus, we have:

1. $\left(F-\mathbb{1}_{S}\right)(x)=F(x)-1$, for any $x \in S$; and

2. $\left(F-\mathbb{1}_{S}\right)(x)=F(x)$, for any $x \in \bar{S}$.

Let $(x, y)$ be a free pair for $F$. The map $F-\mathbb{1}_{\{x, y\}}$ is called an elementary collapse of $F$ or, more precisely, an elementary $d$-collapse of $F$ if $(x, y)$ is a free $d$ pair. Thus, the elementary collapse $F-\mathbb{1}_{\{x, y\}}$ of $F$ is obtained by subtracting 1 to the values of $x$ and $y$. Remark that the elementary collapse $H=F-\mathbb{1}_{\{x, y\}}$ of $F$ is a simplicial stack since we have $F[k]=H[k]$ for any $k \in \mathbb{K} \backslash\{F(x)\}$ and $H[k]$ is an elementary collapse of $F[k]$.

In other words, an elementary collapse of $F$ is a stack obtained from $F$ by decreasing by one the altitudes of the two faces of a free pair for $F$ while leaving unchanged the altitude of any other face.

For instance, the stack shown in Fig. 16a is an elementary 2-collapse of $F$ (Fig. 15a). More precisely, 


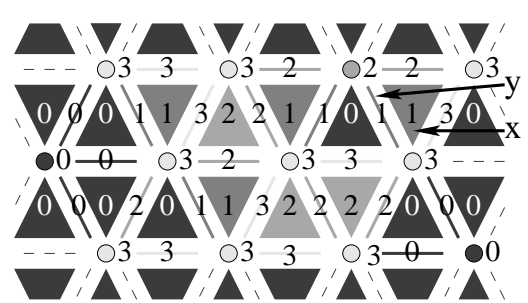

(a)

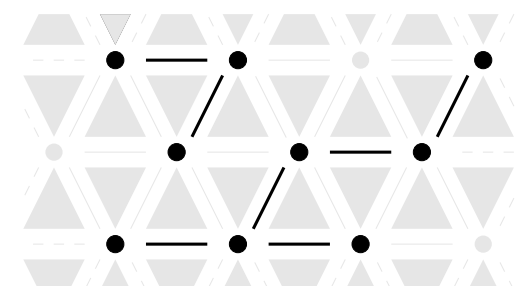

(d)

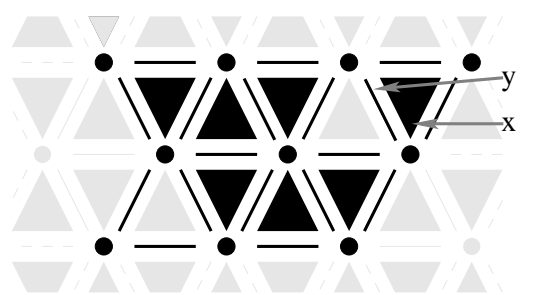

(b)

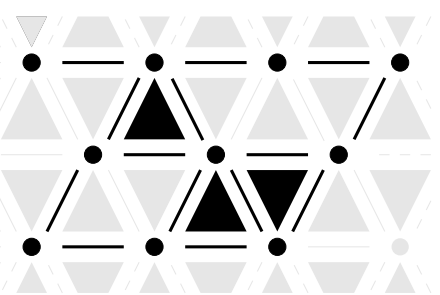

(c)

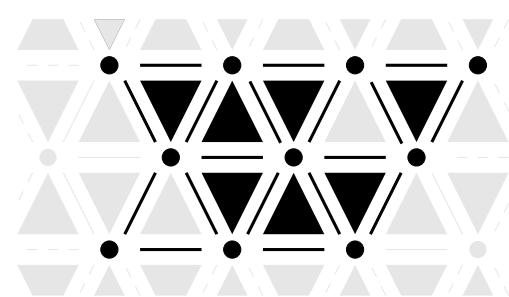

(e)

Fig. 15 (a) Representation of a simplicial stack $F$. The gray level of a face corresponds to its altitude which is also indicted by the superimposed number (the faces with no values are supposed to be at altitude 0 ). (b,c,d) The $k$-section of $F$ for respectively $k=1,2,3$. (e) The minima of $F$ are represented in gray and the divide $\mathcal{D}_{F}$ of $F$ is represented in black.

to obtain this second stack, the values of $x$ and $y$ (see Fig. 15a) have been lowered by one and the pair $(x, y)$ is a free 2-pair for $F$.

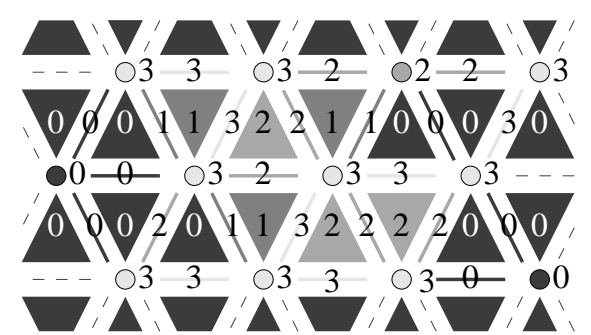

(a)

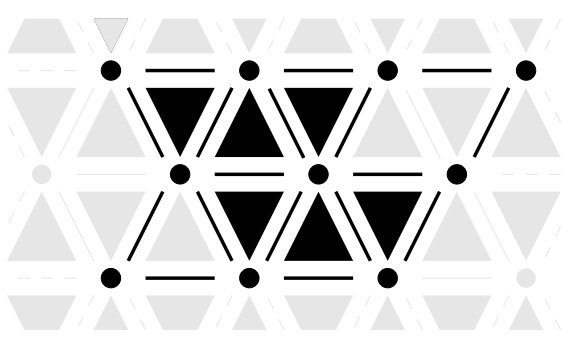

(b)

Fig. 16 A map (a) which is an elementary 2-collapse of the map of Fig. 15a and its minima in gray (b).

Let $H$ be a simplicial stack on $\mathbb{M}$. We say that $H$ is a collapse of $F$ if there exists a collapse sequence from $F$ to $H$, i.e., a sequence of stacks $\left\langle F_{0}, \ldots, F_{\ell}\right\rangle$ such that $F_{0}=F, F_{\ell}=H$ and $F_{i}$ is an elementary collapse of $F_{i-1}, i \in\{1, \ldots, \ell\}$. If each $F_{i}$ is an elementary $d$ collapse of $F_{i-1}$, we also say that $H$ is a $d$-collapse of $F$.
If $H$ is a $d$-collapse of $F$ and if there is no free $d$-pair for $H, H$ is said to be an ultimate $d$-collapse of $F$.

The stack $H$ (Fig. 17a) depicts an ultimate 2collapse of the stack $F$ (Fig. 15a).

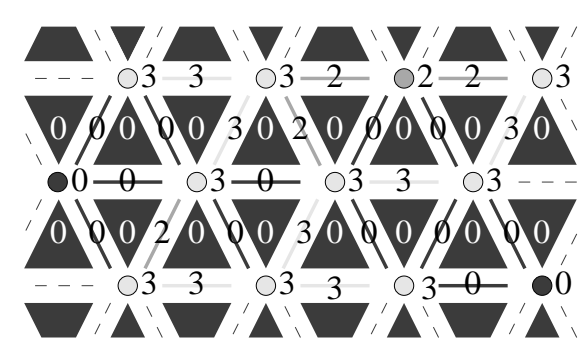

(a)

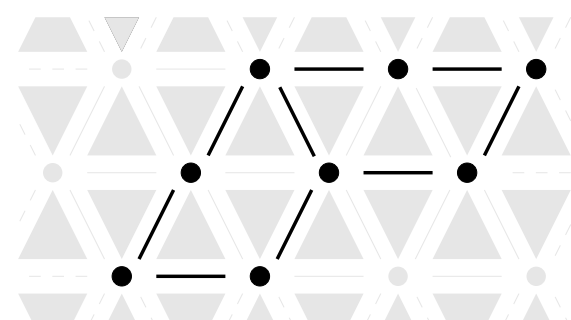

(b)

Fig. 17 (a) An ultimate 2-collapse $H$ of the map $F$ depicted in Fig. 15a. (b) a watershed $X$ of $F$ which is equal to the set of all multiconnected faces of $\bar{M}(H)$.

Remark 23 Note that a stack $H$ is a collapse of $F$ if and only if, for any $k \in \mathbb{K}$, the $k$-section of $H$ is a collapse of the $k$-section of $F$. In this sense, we can say that the operation of collapse in simplicial stacks 
extends the one on simplicial complexes. In particular, if $F$ is the indicator map $\mathbb{1}_{X}$ of a complex $X$ (i.e. $a$ simplicial stack such that the altitude of the faces of $X$ equals 1 and the altitude of the faces in $\bar{X}$ equals 0 ), it is equivalent to consider a collapse of $F$ or to consider the indicator map $\mathbb{1}_{Y}$ of a collapse $Y$ of the complex $X$.

The divide (or support) of a stack $H$, denoted by $\mathcal{D}_{H}$, is the set of all faces of $\mathbb{M}$ which do not belong to any minimum of $H: \mathcal{D}_{H}=\overline{\mathcal{M}_{H}}$. Note that since $\mathcal{M}_{H}$ is a star, by Property $3, \mathcal{D}_{H}$ is a complex.

The divide of the stack of Fig. 15a is shown in black in Fig. 15e.

Let us now establish some interesting properties of collapses of stacks. We will first see (Property 25) that if a stack $H$ is a collapse of $F$, then the divide of $H$ is a collapse (in the binary sense, see Section 3 ) of the divide of $F$. Additionally, we will see that if $H$ does not contain any free $n$-pair, then the divide of $H$ does not contain any free $n$-pair, the converse being also true. Through these two properties, we can see that the collapses of stacks lead to new strategies for obtaining collapses of binary objects. Indeed, if $X \preceq \mathbb{M}$, one can proceed in three steps for obtaining a collapse $Y$ of $X$ : i) compute a stack $F$ such that $\mathcal{D}_{F}=X$; ii) compute a collapse $H$ of $F$; and iii) set $Y$ to the divide $\mathcal{D}_{H}$ of $H$. As we will see formally later on (Theorem 30), this strategy is interesting since the obtained collapses are localized on the "crests" of the map. In this perspective, it is for instance interesting to consider at step ii) a stack $H$ which behaves like a distance function to the object $\bar{X}$ (obtained e.g. by considering the simplicial closure of a Euclidean distance maps on complexes [52]). Indeed, in this case the obtained collapse is localized "as far as possible" from $\bar{X}$ that is, in other words, it is "centered" in $X$.

Lemma 24 Let $(x, y)$ be a free pair for $F$. Let $H$ be the elementary collapse of $F$ such that $H=\left[F-\mathbb{1}_{\{x, y\}}\right]$. Then, $\mathcal{D}_{H}$ is an elementary collapse of $\mathcal{D}_{F}$ that is either equal to $\mathcal{D}_{F}$ or to $\mathcal{D}_{F} \backslash\{x, y\}$.

Proof If $x$ and $y$ do not belong to $\mathcal{M}_{H}$, then it can be easily seen that they do not belong to $\mathcal{M}_{F}$. Thus, we have $\mathcal{D}_{H}=\mathcal{D}_{F}$, which establishes the result.

If $x$ (resp. $y$ ) belongs to a minimum of $H$, it can be easily seen, by definition of a minimum, that $y$ (resp. $x$ ) belongs to the same minimum of $H$ since $y \subseteq x$ and $H(x)=H(y)=k-1$, with $k=F(x)$. Let us then suppose that $x$ and $y$ both belong to $\mathcal{M}_{H}$. Let $M^{\prime}$ be the minimum of $H$ that contains $x$ and $y$. By definition of a minimum and of $H, H\left(M^{\prime}\right)=k-1$ and $M^{\prime}$ is a connected component of $\overline{H[k]}$. By definition of $H, H[k]$ is a collapse of $F[k]$. Thus, by Property $11, \overline{H[k]}$ is an extension of $\overline{F[k]}$. Let $M=M^{\prime} \backslash\{x, y\}$. Since $M$ is a subset of $\overline{F[k]}$ and since $M \subset M^{\prime}$, we deduce that $M$ is a connected component of $\overline{F[k]}$. Since $M^{\prime}$ is a minimum of $H$, we also have $M^{\prime} \cap \overline{H[k-1]}=\emptyset$. Again by definition of $H$, we have $\overline{H[k-1]}=\overline{F[k-1]}$. Thus, since $M \subseteq M^{\prime}$, we deduce that $M \cap \overline{F[k-1]}=\emptyset$, which (together with the fact that $M$ is a connected component of $\overline{H[k]}$ ) implies that $M$ is a minimum of $F$. Since $M^{\prime}$ is a minimum of $H, M^{\prime}$ is a star. Therefore, since $y \in M^{\prime}$, we deduce that $\operatorname{star}(y) \subseteq M^{\prime}$. Hence, $[\operatorname{star}(y) \backslash\{x, y\}] \subseteq \mathcal{M}_{F}$. Then, we deduce that $x$ is the only face of $\mathcal{D}_{F}$ that contains $y$, which is sufficient to prove that $\mathcal{D}_{H}$ is an elementary collapse of $\mathcal{D}_{F}$ and that $\mathcal{D}_{H}=\mathcal{D}_{F} \backslash\{x, y\}$.

The previous property can be verified on the map $F$ (Fig. 15a) and its elementary 2-collapse $H$ (Fig. 16a); the divides of $F$ and $H$ are drawn in black in respectively Fig. 15e and Fig. 16b.

From Lemma 24, we deduce by induction the following result which establishes that the collapses of stacks allow for obtaining collapses of complexes.

Property 25 If $H$ is a collapse of $F$, then $\mathcal{D}_{H}$ is a collapse of $\mathcal{D}_{F}$.

Since collapses and extensions are linked through Property 11, the next corollary follows straightforwardly. It states in particular that the number of minima of a stack is the same as the number of minima of its collapses.

Corollary 26 Let $H$ be a collapse of $F$. Then $\mathcal{M}_{H}$ is an extension of $\mathcal{M}_{F}$.

Property 27 The simplicial stack $F$ contains a free $n$-pair if and only if $\mathcal{D}_{F}$ contains a free $n$-pair.

In order to prove Property 27, we first need to define descending paths.

Definition 28 Let $\pi=\left\langle x_{0}, \ldots, x_{\ell}\right\rangle$ be a path in $\mathbb{M}$. We say that the path $\pi$ is descending (for $F$ ) if, for any $i \in\{1, \ldots, \ell\}, F\left(x_{i}\right) \leqslant F\left(x_{i} \cap x_{i-1}\right) \leqslant F\left(x_{i-1}\right)$.

We say that the path $\pi$ is an $\mathcal{M}$-path for $F$, if $\pi$ is a simple path, if $x_{\ell}$ belongs to $\mathcal{M}_{F}$, and if none of $\left\{x_{i} \mid i<\ell\right\}$ belongs to $\mathcal{M}_{F}$.

Observe that, since $\mathbb{M}$ is finite, from any $n$-face of $\mathbb{M}$, there exists a simple path that is descending for $F$ and whose last element belongs to $\mathcal{M}_{F}$, or, in other words, for any $n$-face of $\mathbb{M}$, there exists an $\mathcal{M}$-path for $F$ that is descending for $F$.

\section{Proof (of Property 27)}

1. Suppose that there exists a free $n$-pair $(x, y)$ for $F$. Let $k=F(x)=F(y)$. Thus, the pair $(x, y)$ is a free $n$-pair for $F[k]$ and we have $\operatorname{dim}(x)=n$ 
and $\operatorname{dim}(y)=n-1$. Since $\mathbb{M}$ is a $n$-pseudomanifold, there exists a unique $n$-face $z$ such that $y \subseteq z$ and $z \neq x$. Hence, since $(x, y)$ is a free $n$-pair for $F[k]$, the face $z$ is not in $F[k]$. Thus, we have $F(z)<k$. Therefore, from the definition of a minimum, it can be seen that $x$ and $y$ do not belong to any minimum of $F$. Thus, the face $x$ is in $\mathcal{D}_{F}$, and, therefore, we have $\operatorname{dim}\left(\mathcal{D}_{F}\right)=n$. Hence, by Property 6 , there exists a free $n$-pair for $\mathcal{D}_{F}$.

2. Suppose now that there exists a free $n$-pair $(x, y)$ for $\mathcal{D}_{F}$. By definition of a free $n$-pair, the dimension of $x$ is $n$. Since $x$ does not belong to any minimum of $F$, there exists a simple path $\pi=\left\langle x_{0}, \ldots, x_{\ell}\right\rangle$ such that $x_{0}=x$, such that $\pi$ is a descending path for $F$, and such that $x_{\ell}$ belongs to a minimum of $F$. Let $i$ be the lowest index in $\{1, \ldots, \ell\}$ such that $x_{i}$ belongs to a minimum of $F$. By definition of a descending path we have $F\left(x_{i}\right) \leqslant$ $F\left(x_{i} \cap x_{i-1}\right) \leqslant F\left(x_{i-1}\right)$. Since, $x_{i-1}$ does not belong to a minimum and since $\mathcal{D}_{F}$ is a complex, we deduce that the $(n-1)$-face $z=x_{i-1} \cap x_{i}$ belongs to $\mathcal{D}_{F}$. Thus, $F\left(x_{i}\right)<F(z)$. On the other hand, since $F$ is a stack and $z \subseteq x_{i-1}$, we must have $F(z) \geqslant F\left(x_{i-1}\right)$. Let $k=F(z)$. From the previous relation, we have $F(z)=F\left(x_{i-1}\right)=k$. Since $\mathbb{M}$ is a $n$-pseudomanifold and $z$ is an $(n-1)$ face, there are exactly two distinct $n$-faces of $\mathbb{M}$ that contain $z$. Hence, as $x_{i}$ and $x_{i-1}$ are two distinct $n$ faces containing $z$, from the underlined relations we deduce that $x_{i-1}$ is the only $n$-face of $F[k]$ which contains $x$. In other words, $\left(x_{i-1}, z\right)$ is a free $n$-pair for $F$.

From the previous result, we derive Property 29 which is an analog of Property 6 for the case of stacks. A consequence of this property is that the divide of any ultimate collapse of $F$ is thin in the sense that its dimension is at most $n-1$.

Property 29 If $\operatorname{dim}\left(\mathcal{D}_{F}\right)=n$, then there exists a free $n$-pair for $F$.

Proof If $\operatorname{dim}\left(\mathcal{D}_{F}\right)=n$, there exists, by Property 6, a free $n$-pair for $\mathcal{D}_{F}$. Hence, by Property 27 , there is also a free $n$-pair for $F$.

Intuitively, one could expect that if a collapse $Y$ of $X=\mathcal{D}_{F}$ is localized on the "crests" of $F$ and if a face $x$ does not belong to $Y$ then there must exist a descending path $\pi$ that starts from $x$ and ends in $\bar{X}=\mathcal{M}_{F}$ without crossing $Y$. The following theorem asserts that the present framework leads to collapses that satisfy this intuitive property. More precisely the next result states that if $H$ is an $n$-collapse of $F$, then the divide $\mathcal{D}_{H}$ is localized on crests.

Before stating Theorem 30, let us recall that, for any stack $H$, we have $\mathcal{M}_{H}=\overline{\mathcal{D}_{H}}$.

Theorem 30 Let $H$ be an $n$-collapse of $F$. Let $x_{0}$ be an $n$-face of $\mathbb{M}$.

1. If $x_{0} \in \mathcal{M}_{H}$, then there exists a path $\pi=$ $\left\langle x_{0}, \ldots, x_{\ell}\right\rangle$ such that:

$-\pi$ is a path in $\mathcal{M}_{H}$; and

- $\pi$ is a descending path for $F$; and

- $x_{\ell}$ belongs to $\mathcal{M}_{F}$.

2. If $x_{0} \in \mathcal{D}_{H}$, then any $\mathcal{M}$-path $\pi=\left\langle x_{0}, \ldots, x_{\ell}\right\rangle$ for $H$ that is descending for $H$ is also descending for $F$.

The remaining part of the section is devoted to the proof of Theorem 30 .

Lemma 31 Let $H$ be an elementary $n$-collapse of $F$. Let $\pi=\left\langle x_{0}, \ldots, x_{\ell}\right\rangle$ be an $\mathcal{M}$-path for $H$ which is descending for $H$. Then $\pi$ is a descending path for $F$.

Proof By definition of an elementary $n$-collapse, there exists a free $n$-pair $(x, y)$ for $F$ such that $H=[F-$ $\left.\mathbb{1}_{\{x, y\}}\right]$. In order to prove Lemma 31 we are going to distinguish three cases.

Case 1. Suppose that for any $i$ in $\{0, \ldots, \ell\} x_{i} \neq x$. Then, for any $i \in\{0, \ldots, \ell\}, F\left(x_{i}\right)=H\left(x_{i}\right)$. Since $\mathbb{M}$ is an $n$-pseudomanifold, for any $j \in\{1, \ldots, \ell\}, x_{j-1}$ and $x_{j}$ are the only two faces of $\mathbb{M}$ which contain $z_{j}=$ $x_{j-1} \cap x_{j}$. Since $x \neq x_{j-1}$, since $x \neq x_{j}$ and since $y \subseteq x$, we deduce that $z_{j} \neq y$. Thus, by hypothesis, we also have $F\left(z_{j}\right)=H\left(z_{j}\right)$ for any $j \in\{1, \ldots, \ell\}$, which together with the underlined relation proves that $\pi$ is descending for $F$.

Case 2. Suppose now that there exists $i \in$ $\{0, \ldots, \ell-1\}$ such that $x_{i}=x$. We denote by $w$ the $(n-1)$-face that equals $x_{i} \cap x_{i+1}$. As $w \subseteq x_{i}$ and as $F$ is a stack, we deduce that $F(w) \geqslant F\left(x_{i}\right)$. Since $\pi$ is a descending path for $H$, we also have $H\left(x_{i}\right) \geqslant H(w)$. Thus, as $H\left(x_{i}\right)=F\left(x_{i}\right)-1$, we have $H(w)<F(w)$. Hence, we deduce that $H(w) \neq F(w)$. Since $w$ is an $(n-1)$-face, this implies $w=y$. Hence, $F(w)=F\left(x_{i}\right)$ and $H(w)=k-1$. As $w \subseteq x_{i+1}$ and as $F$ is a stack, we also deduce that $F\left(x_{i+1}\right) \leqslant F(w)$. If $i=0$, the underlined relations and the fact that $F(z)=H(z)$ for any $z \in \mathbb{M} \backslash\{x, y\}$ imply that $\pi$ is descending for $F$ which completes the proof of Lemma 31 in case 2 . Let us now assume that $i>0$ and denote by $w^{\prime}$ the $(n-1)$-face that equals $x_{i-1} \cap x_{i}$. As $w^{\prime} \subseteq x_{i}$ and as $F$ is a stack, we have $F\left(w^{\prime}\right) \geqslant F\left(x_{i}\right)$. Since $w^{\prime} \neq y$ and since $x_{i-1} \neq x$, we have $F\left(w^{\prime}\right)=H\left(w^{\prime}\right)$ and $F\left(x_{i-1}\right)=H\left(x_{i-1}\right)$. Since $\pi$ is descending for $H, H\left(w^{\prime}\right) \leqslant H\left(x_{i-1}\right)$. Thus, $F\left(w^{\prime}\right) \leqslant F\left(x_{i-1}\right)$. From the underlined relations 
and from the fact that $F(z)=H(z)$ for any $z \in$ $\mathbb{M} \backslash\{x, y\}$, we deduce that $\pi$ is a descending path for $F$.

Case 3. Suppose finally that $x_{\ell}=x$. If $\ell=0$, the proof is trivial. Let us now assume that $\ell>0$ and denote by $w$ the $(n-1)$-face which is equal to $x_{\ell-1} \cap x_{\ell}$. Since $\pi$ is an $\mathcal{M}$-path for $H, x_{\ell-1} \in \mathcal{D}_{H}$. Since $\mathcal{D}_{H}$ is a complex (by definition of a stack), $w \subseteq x_{\ell-1}$ implies $w \in \mathcal{D}_{H}$. Since $\pi$ is an $\mathcal{M}$-path for $H, x_{\ell} \in$ $\mathcal{M}_{H}$. Hence, by definition of a minimum, we must have $H(w)>H\left(x_{\ell}\right)$. Thus, since $H(x)=H(y)$ by construction of $H$, we deduce that $w \neq y$, Thus, we have $F(w)=H(w)$ and therefore, we also have $F(w) \geqslant$ $F\left(x_{\ell}\right)$. We can also deduce easily that, for any $i$ in $\{0, \ldots, \ell-1\}, F\left(x_{i}\right)=H\left(x_{i}\right)$ and $F\left(x_{i+1} \cap x_{i}\right)=$ $H\left(x_{i+1} \cap x_{i}\right)$. Therefore, the path $\pi$ is descending for $F$.

Lemma 32 Let $(x, y)$ be a free $n$-pair for $F$. Let $H$ be the elementary collapse of $F$ such that $H=\left[F-\mathbb{1}_{\{x, y\}}\right]$. Let $\pi=\left\langle x_{0}, \ldots, x_{\ell}\right\rangle$ be an $\mathcal{M}$-path for $H$ that is descending for $H$.

1. If $x_{\ell} \neq x$, then $\pi$ is an $\mathcal{M}$-path for $F$ that is descending for $F$.

2. If $x_{\ell}=x$, then there exists an $n$-face $z$ such that $\pi^{\prime}=\left\langle x_{0}, \ldots, x_{\ell}, z\right\rangle$ is an $\mathcal{M}$-path for $F$ that is descending for $F$ and $\left\langle x_{\ell}, z\right\rangle$ is a path in $\mathcal{M}_{H}$.

Proof 1. Suppose that $x_{\ell} \neq x$. By Lemma 24, we have either $\mathcal{D}_{H}=\mathcal{D}_{F}$ or $\mathcal{D}_{H}=\mathcal{D}_{F} \backslash\{x, y\}$, and, thus, we have either $\mathcal{M}_{F}=\mathcal{M}_{H}$ or $\mathcal{M}_{F}=\mathcal{M}_{H} \backslash\{x, y\}$. Since $\pi$ is an $\mathcal{M}$-path for $H$, the face $x_{\ell}$ belongs to $\mathcal{M}_{H}$ and the faces $x_{0}, \ldots, x_{\ell-1}$ belong to $\mathcal{D}_{H}$. Thus, the faces $x_{0}, \ldots, x_{\ell-1}$ also belong to $\mathcal{D}_{F}$. Furthermore, as $x_{\ell} \neq x$ and as $x_{\ell} \neq y\left(\operatorname{since} \operatorname{dim}\left(x_{\ell}\right)=n\right.$ and $\operatorname{dim}(y)=n-1)$, the face $x_{\ell}$ is in $\mathcal{M}_{F}$. Therefore, the path $\pi$ is an $\mathcal{M}$-path for $F$. By Lemma 31, the path $\pi$ is descending for $F$.

2. Let us now suppose that $x_{\ell}=x$. Let $k=F(x)=$ $F(y)$. Then, $H(x)=H(y)=k-1$. By hypothesis, $(x, y)$ is a free $n$-pair for $F[k]$. Thus, $x$ is the only $n$ face that contains $y$ and belongs to $F[k]$. Therefore, since $\mathbb{M}$ is an $n$-pseudomanifold, there exists a unique $n$-face $z$ which belongs to $\overline{F[k]}$ and contains $y$. We then have the relations $F(x) \geqslant F(y)>F(z)$. Note also that $y=x \cap z$. By Lemma 31, the path $\pi$ is descending for $F$. Thus, we deduce that the sequence $\pi^{\prime}=\left\langle x_{0}, \ldots, x_{\ell}, z\right\rangle$ is a path that is descending for $F$.

We will establish that $\pi^{\prime}$ is an $\mathcal{M}$-path for $F$. Since $F(z)<F(y)$ and since $z$ contains $y$, the $(n-1)$ face $y$ does not belong to $\mathcal{M}_{F}$. Hence, as $F(x) \geqslant F(y)$ and as $x$ contains $y$, the $n$-face $x$ does not belong to $\mathcal{M}_{F}$ either. But, since $\pi$ is an $\mathcal{M}$-path for $H$, the face $x=x_{\ell}$ belongs to $\mathcal{M}_{H}$. Hence, by Lemma 24, we deduce that $\mathcal{D}_{H}$ is an elementary collapse of $\mathcal{D}_{F}$ that is equal to $\mathcal{D}_{F} \backslash\{x, y\}$. Thus, the pair $(x, y)$ is a free $n$-pair for $\mathcal{D}_{F}$. Hence, the simplex $x$ is the only $n$-face of $\mathcal{D}_{F}$ that contains $y$. Thus, the $n$-face $z$ belongs to $\overline{\mathcal{D}_{F}}=\mathcal{M}_{F}$. As $\pi$ is an $\mathcal{M}$ path for $H$, the faces $x_{0}, \ldots, x_{\ell-1}$ belong to $\mathcal{D}_{H}$ and, as $\mathcal{D}_{H}=\mathcal{D}_{F} \backslash\{x, y\}$, the faces $x_{0}, \ldots, x_{\ell-1}$ also belong to $\mathcal{D}_{F}$. Hence, the sequence $\pi^{\prime}$ is an $\mathcal{M}$-path for $F$. Since, $\mathcal{D}_{H}=\mathcal{D}_{F} \backslash\{x, y\}$, we have $\mathcal{M}_{H}=\mathcal{M}_{F} \cup$ $\{x, y\}$. Thus, $x$ and $y$ belong to $\mathcal{M}_{H}$. Furthermore, since $z$ belongs $\mathcal{M}_{F}, z$ also belongs to $\mathcal{M}_{H}$. Thus, the sequence $\langle x, z\rangle$ is a path in $\mathcal{M}_{H}$. Hence, the three underlined relations complete the proof of Lemma 32.

Proof (of Theorem 30, by induction) If $H=F$, then the result is trivial. Let us now assume that $H \neq F$. Thus, there exists a collapse sequence $\left\langle F=F_{0}, \ldots, F_{\ell}=H\right\rangle$ from $F$ to $H$. As induction hypothesis, we assume that Theorem 30 holds true for $F_{i}=I$, with $i \in\{0, \ldots, \ell-$ $1\}$. In order to complete the proof by induction, it is sufficient to prove, under this induction hypothesis, that Theorem 30 also holds true for $G=F_{i+1}$.

Since $G$ is an elementary collapse of $I$, there exists a free $n$-pair $(x, y)$ for $I$ such that $G=\left[I-\mathbb{1}_{\{x, y\}}\right]$. Let $x_{0}$ be any $n$-face in $\mathbb{M}$. We distinguish three cases.

1. We first consider the case where $x_{0}$ belongs to $\mathcal{M}_{G}$ and $x_{0} \neq x$. Then, the trivial path $\left\langle x_{0}\right\rangle$ is an $\mathcal{M}$ path for $G$. By Lemma 32.1, the path $\left\langle x_{0}\right\rangle$ is also an $\mathcal{M}$-path for $I$ that is descending of $I$. Thus $x_{0}$ belongs to $\mathcal{M}_{I}$. Therefore, by induction hypothesis, there exists a path $\pi$ in $\mathcal{M}_{I}$ that is descending for $F$ and that ends in $\mathcal{M}_{F}$. By Corollary 26, we have $\mathcal{M}_{I} \sqsubseteq \mathcal{M}_{G}$. Thus, we deduce that $\pi$ is also a path in $\mathcal{M}_{G}$.

2. We now consider the case where $x_{0}$ belongs to $\mathcal{M}_{G}$ and $x_{0}=x$. Then, the trivial path $\left\langle x_{0}\right\rangle$ is an $\mathcal{M}$ path for $G$ that is descending for $G$. By Lemma 32.2, there exists a $n$-face $x_{1}$ such that $\left\langle x_{0}, x_{1}\right\rangle$ is an $\mathcal{M}$-path for $I$ that is descending for $I$ and such that $\left\langle x_{0}, x_{1}\right\rangle$ is a path in $\mathcal{M}_{G}$. By induction hypothesis, the path $\left\langle x_{0}, x_{1}\right\rangle$ is descending for $F$ and there exists a path $\left\langle x_{1}, \ldots, x_{k}\right\rangle$ in $\mathcal{M}_{I}$ that is descending for $F$ and that ends in $\mathcal{M}_{F}$. Thus, as $\mathcal{M}_{I} \sqsubseteq \mathcal{M}_{G}$ by Corollary 26, we deduce that $\left\langle x_{0}, x_{1}, \ldots, x_{k}\right\rangle$ is a path in $\mathcal{M}_{G}$ that is descending for $F$ and that ends in $\mathcal{M}_{F}$.

3. We finally consider the case where $x_{0}$ belongs to $\mathcal{D}_{G}$. Let $\pi=\left\langle x_{0}, \ldots, x_{k}\right\rangle$ be any $\mathcal{M}$-path for $G$ that is descending for $G$. If $x_{k} \neq x$, then, from Lemma 32.1 , the path $\pi$ is a descending $\mathcal{M}$ path for $I$, and therefore, by induction hypothesis, the path $\pi$ is also descending for $F$. If $x_{k}=$ $x$, from Lemma 32.2 , there exists a $n$-face $x_{k+1}$ 
such that $\pi^{\prime}=\left\langle x_{0}, \ldots, x_{k}, x_{k+1}\right\rangle$ is an $\mathcal{M}$-path for $I$ descending for $I$. By induction hypothesis, the path $\pi^{\prime}$ is also descending for $F$. Thus, the path $\pi$ is also descending for $F$, which completes the proof of Theorem 30 .

\section{Watersheds}

Based on the drop of water principle [23,24], we propose in this section a definition of a watershed in a pseudomanifold. Then, we present the two main results of this paper (namely Theorems 34 and 36): the first one establishes a strong link between the watersheds of $F$ and the homotopy type (i.e., the topology) of the divide $\mathcal{D}_{F}$ of $F$, and the second one establishes the equivalence between the watersheds of $F$ and the sets of multiconnected faces obtained from the ultimate $n$ collapses of $F$.

Intuitively, the regions, also called catchment basins, delimited by a watershed constitute an extension of the minima and they are separated by a cut from which a drop of water can flow down towards distinct minima.

Definition 33 Let $X \preceq \mathbb{M}$ be a cut for $\mathcal{M}_{F}$. We say that $X$ is a watershed of $F$ if, for any $x \in X$, there exist two paths $\pi_{1}=\left\langle x_{0}, \ldots, x_{\ell}\right\rangle$ and $\pi_{2}=\left\langle y_{0}, \ldots, y_{m}\right\rangle$ in $\bar{X}$ such that:

$-x \subseteq x_{0}$ and $x \subseteq y_{0}$; and

- $\pi_{1}$ and $\pi_{2}$ are two descending paths for $F$; and

- $x_{\ell}$ and $y_{m}$ are simplices of two distinct minima of $F$.

For instance, the set of black faces in Fig. $17 \mathrm{~b}$ is a watershed of the map in Fig. 15a.

The main result of this section (namely Theorem 36) establishes the equivalence between the watersheds of $F$ and the cuts by collapse (see Definition 35) of $F$. The definition of a cut by collapse is constructive and, following this constructive definition, one can always obtain a cut by collapse from a stack. Thus, Theorem 36 also establishes that, whatever the stack $F$, there always exits a watershed of $F$. However, as in the case of weighted graphs, there may exist several distinct watersheds of the same stack $F$. This is in particular the case when the map $F$ has two minima which are separated by a plateau. The position of the watershed contours on the plateaus is the subject of many discussions which are beyond the scope of this paper (see e.g., $[42,49]$ ).

Any watershed of $F$ is by definition a complex and a cut for $\mathcal{M}_{F}$. Thus, the following result can be straightforwardly deduced from Property 13 and Theorem 15.

Theorem 34 The two following statements hold true.
1. Any watershed of $F$ is either empty or is a pure ( $n-$ 1)-complex.

2. Any watershed of $F$ is a cut by collapse of $\mathcal{M}_{F}$.

The first part of the previous theorem establishes that the watershed of any stack is thin in the sense that its dimension is always lower than the one of the space. The second part of the theorem asserts that any watershed of $F$ is made of the closed contours (in the sense of Definition 14) of an ultimate collapse of $X=$ $\mathcal{D}_{F}$, establishing a direct link between the watersheds of $F$ and the homotopy type of $X$. We remind that these two results hold true in any $n$-pseudomanifold, whatever the dimension $n \in \mathbb{N}$.

As far as we know, in the literature, there is only one context where some watersheds satisfy simultaneously two statements similar to those of Theorem 34. More precisely, given a continuous open subset $X$ of $\mathbb{R}^{n}$, there is a link between the watershed $W(X)$ of the Euclidean distance transform $D_{X}$ of $X$ and the homotopy type of $X$. Furthermore, the watershed $W(X)$ satisfies a thinness property. In fact, the three following properties hold true in this continuous setting.

1. The watershed of $D_{X}$ is made of the closed contours of the medial axis $M A(X)$ of $X$ (see $[9,10]$ for the definition of medial axis, and see in particular the work of L. Najman and M. Schmitt in [42] for a proof of this statement).

2. The set $X$ and its medial axis $M A(X)$ have the same homotopy type (the first study of this question was provided by G. Matheron in [38,39], and a proof of the statement was given by A. Rivière in $[46,47]$ and by A. Lieutier in [35]).

3. The interior of the skeleton $M A(X)$ (and hence also the interior of the watershed $W(X))$ is empty $[38,39]$ and furthermore $M A(X)$ and $W(X)$ are Lebesgue negligible [46,47].

Furthermore, a result similar to Theorem 34 has not been investigated in a discrete setting. In particular, in digital topology, there is no such a straightforward relation between homotopic skeletons and a notion of watershed. Moreover, in general, a watershed can be arbitrary thick $[21,25]$.

In the remaining part of this section, a second strong link between watershed and homotopy is established by Theorem 36 . This result states i) that any ultimate $n$ collapse of the stack $F$ directly leads to a watershed of $F$, and ii) that any watershed of $F$ can be directly obtained from an ultimate $n$-collapse of $F$. In fact, ultimate $n$-collapses can be computed in linear time (using the same thinning strategies as those presented in [24]). Therefore, Theorem 36 furthermore 
indicates an efficient algorithmic strategy for computing watersheds.

On one hand, the divide of an ultimate $n$-collapse $H$ of $F$ is located on the "crests" of $F$ in the sense of Theorem 30. On the other hand, we can say intuitively that a watershed of $F$ corresponds to the "closed contours" located on the "crests" of $F$. Hence, a desirable property is that the watersheds of $F$ correspond to the "closed contours" of the divide of the ultimate $n$-collapses of $F$. The following theorem asserts that this intuitive property is indeed true in the framework presented in this paper.

Definition 35 (Cut by collapse for a stack) Let $X \preceq$ M. We say that $X$ is a cut by collapse for $F$ if there exists an ultimate $n$-collapse $H$ of $F$ such that $X$ is the simplicial closure of the set of all $(n-1)$-faces that are multiconnected for $\mathcal{D}_{H}$.

The next theorem, which is one of our main results, generalizes Theorem 15 to the case of stacks. It establishes a deep link between watersheds and homotopy.

Theorem 36 Let $X \preceq \mathbb{M}$. The set $X$ is a watershed of $F$ if and only if $X$ is a cut by collapse for $F$.

The remaining part of this section is devoted to the proof of Theorem 36. To this end, we first prove the backward implication of the theorem (Property 39) and then the forward implication (Property 43).

Lemma 37 Let $H$ be an elementary $n$-collapse of $F$ and let $X \preceq \mathbb{M}$. If $X$ is a watershed of $H$, then $X$ is a watershed of $F$.

Proof By Corollary 26, $\mathcal{M}_{H}$ is an extension of $\mathcal{M}_{F}$. Since $X$ is a watershed of $H$, the complex $X$ is a cut for $\mathcal{M}_{H}$, and then the star $\bar{X}$ is an extension of $\mathcal{M}_{H}$. Thus, by Remark 9 , the star $\bar{X}$ is an extension of $\mathcal{M}_{F}$. Let $x$ be any face of $X$. By definition of a watershed, there exist two paths $\pi_{1}=\left\langle x_{0}, \ldots, x_{\ell}\right\rangle$ and $\pi_{2}=\left\langle y_{0}, \ldots, y_{m}\right\rangle$ in $\bar{X}$ such that:

$-x \subseteq x_{0}$ and $x \subseteq y_{0}$; and

$-\pi_{1}$ and $\pi_{2}$ are two descending paths for $H$; and

- $x_{\ell}$ and $y_{m}$ are simplices of two distinct minima of $H$.

Thus, there exist $i$ and $j$ in $\{0, \ldots, \ell\}$ and $\{0, \ldots, m\}$, respectively, such that $\left\langle x_{0}, \ldots, x_{i}\right\rangle$ and $\left\langle y_{0}, \ldots, y_{j}\right\rangle$ are two $\mathcal{M}$-paths for $H$ that are descending for $H$.

Let us consider the path $\left\langle x_{0}, \ldots, x_{i}\right\rangle$ (resp. $\left.\left\langle y_{0}, \ldots, y_{j}\right\rangle\right)$. From Lemma 32,

1. either $\left\langle x_{0}, \ldots, x_{i}\right\rangle$ (resp. $\left.\left\langle y_{0}, \ldots, y_{j}\right\rangle\right)$ is an $\mathcal{M}$-path for $F$ that is descending for $F$, and in this case we set $\pi_{1}^{\prime}=\left\langle x_{0}, \ldots, x_{i}\right\rangle$ (resp. $\left.\pi_{2}^{\prime}=\left\langle y_{0}, \ldots, y_{j}\right\rangle\right)$;
2. or there exists $x^{\prime}$ (resp. $y^{\prime}$ ) such that $\pi_{1}^{\prime}=$ $\left\langle x_{0}, \ldots, x_{i}, x^{\prime}\right\rangle\left(\operatorname{resp} . \pi_{2}^{\prime}=\left\langle y_{0}, \ldots, y_{j}, y^{\prime}\right\rangle\right)$ is an $\mathcal{M}$ path for $F$, that is descending for $F$, with $\left\langle x_{i}, x^{\prime}\right\rangle$ (resp. $\left.\left\langle y_{j}, y^{\prime}\right\rangle\right)$ in $\mathcal{M}_{H}$.

In the second case, as $\left\langle x_{i}, x^{\prime}\right\rangle$ (resp. $\left.\left\langle y_{j}, y^{\prime}\right\rangle\right)$ is, by Lemma 32 , a path in $\mathcal{M}_{H}$ and as $\mathcal{M}_{H} \subseteq \bar{X}$, we deduce that $\pi_{1}^{\prime}$ (resp. $\pi_{2}^{\prime}$ ) is a path in $\bar{X}$. In any of cases 1 and $2, \pi_{1}^{\prime}$ (resp. $\pi_{2}^{\prime}$ ) is a path in $\bar{X}$ that is descending for $F$. Furthermore, since $\bar{X}$ is an extension of $\mathcal{M}_{F}$ and since $x_{\ell}$ and $y_{m}$ belong to two distinct connected components of $\bar{X}$, we deduce that the last faces of the paths $\pi_{1}^{\prime}$ and $\pi_{2}^{\prime}$ belong to two distinct minima of $F$. $\preceq$ Thus, $X$ is a watershed of $F$. $\square$

Therefore, the following lemma is established by induction.

Lemma 38 Let $H$ be an $n$-collapse of $F$, then any watershed of $H$ is a watershed of $F$.

Property 39 Any cut by collapse of $F$ is a watershed of $F$.

Proof Let $X$ be a cut by collapse of $F$. Then, there exists an $n$-collapse $H$ of $F$ such that $X$ is the simplicial closure of the set of all $(n-1)$-faces that are multiconnected for $\mathcal{D}_{H}$. Since $H$ does not contain any free $n$-pair, by Property $27, \mathcal{D}_{H}$ does not contain any free $n$-pair and thus, $\operatorname{dim}\left(\mathcal{D}_{H}\right)=n-1$ by Property 6 . Thus, $X$ is the only cut by collapse of $\mathcal{M}_{H}$. Therefore, by Theorem $15, X$ is the only cut for $\mathcal{M}_{H}$. Let $x$ be any face of $X$, there exists an $(n-1)$-face of $X$ that contains $x$ and that is multiconnected for $\mathcal{D}_{H}$. Thus, there exist two $n$-faces $x_{0}$ and $y_{0}$ that include $x$ and that belong to two distinct connected components of $\overline{\mathcal{D}_{H}}=\mathcal{M}_{H}$. Hence, we have $x \subseteq x_{0}, x \subseteq y_{o}$, and $\left\langle x_{0}\right\rangle$ and $\left\langle y_{0}\right\rangle$ are two descending paths for $H$ that ends in two distinct minima of $H$. Thus, the complex $X$ is a watershed of $H$. Then, by Lemma $38, X$ is also a watershed of $F$

Lemma 40 Let $X$ be a watershed of $F$. Then, for any $n$-face $x$ of $\mathbb{M}$, there exists a path in $\bar{X}$ from $x$ to an $n$-face of $\mathcal{M}_{F}$, that is a descending path for $F$.

Proof Let $x_{0}$ be any $n$-face of $\mathbb{M}$. Since $\mathbb{M}$ is finite, there exists a path $\pi=\left\langle x_{0}, \ldots, x_{\ell}\right\rangle$ in $\mathbb{M}$ that is descending for $F$ and such that $x_{\ell} \in \mathcal{M}_{F}$. If $\pi$ is a path in $\bar{X}$, the proof is done. Suppose now that $\pi$ is not a path in $\bar{X}$. As $X$ is a watershed of $F, X$ is a cut for $\mathcal{M}_{F}$. We suppose that $X \neq \emptyset$, the property obviously holds true otherwise. Thus, Property 13 implies that the dimension of $X$ equals $n-1$. Then, it follows that there exists an index $i \in\{1, \ldots, \ell\}$ such that $x_{i-1} \cap x_{i} \in X$. Let $i$ be the lowest such index and let $z=x_{i-1} \cap x_{i}$. 
By definition of a path, $z$ is an $(n-1)$-face. Therefore, since $\mathbb{M}$ is a $n$-pseudomanifold, $x_{i-1}$ and $x_{i}$ are the only two $n$-faces that contain $z$. Thus, since $X$ is a watershed of $F$, there exists a path $\left\langle y_{0}, \ldots, y_{m}\right\rangle$ in $\bar{X}$ that is descending for $F$ and such that $y_{m} \in \mathcal{M}_{F}$ and $y_{0}=x_{i-1}$. Then, the path $\left\langle x_{0} \ldots, x_{i-1}, y_{1}, \ldots, y_{m}\right\rangle$ is a path in $\bar{X}$ that is descending for $F . \square$

Lemma 41 Let $X$ be a watershed of $F$. If the dimension of $\mathcal{D}_{F}$ equals $n$, then there exists a free $n$-pair $(x, y)$ for $F$ such that $x$ and $y$ are in $\bar{X}$.

Proof Since the dimension of $\mathcal{D}_{F}$ equals $n$, there exists an $n$-face $x_{0} \in \mathcal{D}_{F}$. Since $X$ is a watershed of $F$, by Lemma 40 , there exists a path $\pi=\left\langle x_{0}, \ldots, x_{\ell}\right\rangle$ in $\bar{X}$ that is descending for $F$ and such that $x_{\ell} \in \mathcal{M}_{F}$. Let $i$ be the lowest index in $\{0, \ldots, \ell\}$ such that $x_{i} \in$ $\mathcal{M}_{F}$. Since $x_{0} \in \mathcal{D}_{F}, i>0$. Let $y$ be the $(n-1)$ face defined by $y=x_{i-1} \cap x_{i}$ and let $k=F(y)$. Thus $y \in F[k]$. Since $\mathcal{D}_{F}$ is a complex, $x_{i-1} \in \mathcal{D}_{F}$, and $y \subseteq x_{i-1}$, we deduce that $F(y) \geqslant F\left(x_{i-1}\right)$ (by definition of a stack) and thus that $y \in \mathcal{D}_{F}$. Hence, as $x_{i} \in \mathcal{M}_{F}, F(y)>F\left(x_{i}\right)$, that is, in terms of sections, $x_{i} \notin F[k]$. On the other hand, since $\pi$ is descending for $F$, we have $F\left(x_{i-1}\right) \geqslant$ $F(y)$, that is, in terms of sections, $x_{i-1} \in F[k]$. As $\mathbb{M}$ is an $n$-pseudomanifold and as the dimension of $y$ is $n-1, x_{i}$ and $x_{i-1}$ are the only two $n$-faces of $\mathbb{M}$ that contain $y$. Then, from the underlined relations, we deduce that $\left(x_{i-1}, y\right)$ is a free $n$-pair for $F$. Furthermore, as $\pi$ is a path in $\bar{X}, x_{i-1}$ and $y$ are in $\bar{X}$.

Lemma 42 Let $X$ be a watershed of $F$. Let $(x, y)$ be a free $n$-pair for $F$ such that $x$ and $y$ are in $\bar{X}$, and let $H$ be the elementary $n$-collapse of $F$ defined by $H=\left[F-\mathbb{1}_{\{x, y\}}\right]$. Then, $X$ is a watershed of $H$.

Proof Since $H$ is an elementary $n$-collapse of $F$, by Corollary 26, the star $\mathcal{M}_{H}$ is an extension of $\mathcal{M}_{F}$ and, thus, that $\mathcal{M}_{F} \sqsubseteq \mathcal{M}_{H}$. Furthermore, by Lemma 24 , we deduce that $\mathcal{M}_{H} \sqsubseteq \mathcal{M}_{F} \cup\{x, y\}$. Since $X$ is a watershed of $F$, the $\operatorname{star} \bar{X}$ is an extension of $\mathcal{M}_{F}$ and thus we have $\mathcal{M}_{F} \sqsubseteq \bar{X}$. As $x$ and $y$ both belong to $\bar{X}$, we also have $\mathcal{M}_{F} \cup\{x, y\} \sqsubseteq \bar{X}$, and therefore we deduce that $\mathcal{M}_{H} \sqsubseteq \bar{X}$. From the underlined relations and Property 10, we deduce that $\bar{X}$ is an extension of $\mathcal{M}_{H}$. Hence, $X$ is a cut for $\mathcal{M}_{H}$.

Let $x^{\prime}$ be any element in $X$. As $X$ is a watershed of $F$, there exist two paths $\pi_{1}=\left\langle x_{0}, \ldots, x_{\ell}\right\rangle$ and $\pi_{2}=$ $\left\langle y_{0}, \ldots, y_{m}\right\rangle$ in $\bar{X}$ such that:

$-x^{\prime} \subseteq x_{0}$ and $x^{\prime} \subseteq y_{0}$; and

- $\pi_{1}$ and $\pi_{2}$ are two descending paths for $F$; and

$-x_{\ell}$ and $y_{m}$ belong to two distinct minima of $F$.
Since $\mathcal{M}_{H}$ is an extension of $\mathcal{M}_{F}$, in order to complete the proof of Lemma 42 , it is sufficient to show that there exist two paths $\pi_{1}^{\prime}$ and $\pi_{2}^{\prime}$ such that:

- the first face of $\pi_{1}^{\prime}$ (resp. $\pi_{2}^{\prime}$ ) includes $x^{\prime}$

- $\pi_{1}$ and $\pi_{2}$ are descending for $H$;

- the last face of $\pi_{1}^{\prime}$ (resp. of $\pi_{2}^{\prime}$ ) belong to the same minimum of $H$ as $x_{\ell}$ (resp. $y_{m}$ ).

If $\pi_{1}$ and $\pi_{2}$ satisfy these three properties, then the proof is done.

Let us now assume that $\pi_{1}$ does not satisfy these three properties. Exactly the same arguments hold true if one assumes that $\pi_{2}$ does not satisfy these properties. As $\mathcal{M}_{H}$ is an extension of $\mathcal{M}_{F}$, it can be seen that necessarily $\pi_{1}$ is not descending for $H$. Thus, there exists $i \in\{1, \ldots, \ell\}$ such that the path $\left\langle x_{0}, \ldots, x_{i-1}\right\rangle$ is descending for $H$ and the path $\left\langle x_{0}, \ldots, x_{i}\right\rangle$ is not. Thus, the inequation $H\left(x_{i}\right) \leqslant$ $H\left(x_{i} \cap x_{i-1}\right) \leqslant H\left(x_{i-1}\right)$ is false. Since $x_{i-1} \cap x_{i} \subseteq x_{i}$ and since $H$ is a stack we have $H\left(x_{i}\right) \leqslant H\left(x_{i} \cap x_{i-1}\right)$. Thus, we have $H\left(x_{i} \cap x_{i-1}\right)>H\left(x_{i-1}\right)$. Since $\pi_{1}$ is descending for $F, F\left(x_{i-1} \cap x_{i}\right) \leqslant F\left(x_{i-1}\right)$. Hence, by definition of $H$, we deduce that $H\left(x_{i-1}\right)<F\left(x_{i-1}\right)$. Thus, we have $x_{i-1}=x$. As $(x, y)$ is a free $n$ pair for $F, y$ is an $(n-1)$-face included in $x$. Since $\mathbb{M}$ is an $n$-pseudomanifold, there exist a unique $n$ face $z_{0}$ such that $z_{0} \cap x_{i-1}=y$. Since $H$ is a stack, $H\left(z_{0}\right) \leqslant H(y)$. As $z_{0} \neq x$ and $z_{0} \neq y$, we have $\overline{H\left(z_{0}\right)=F\left(z_{0}\right)}$ by definition of $H$. Thus, we have $F\left(z_{0}\right) \leqslant H(y)$. Furthermore, as $H(y)=F(y)-1$, we deduce that $F(y)>F\left(z_{0}\right)$. We also have $F(x)>$ $F\left(z_{0}\right)$ since $F(x)=F(y)$. By Lemma 40, there exists a path $\pi^{\prime}=\left\langle z_{0}, \ldots, z_{k}\right\rangle$ in $\bar{X}$ descending for $F$ and such that $z_{k} \in \mathcal{M}_{F}$. Thus, since $F\left(z_{0}\right)<F(x)$, for any $j \in\{1, \ldots, k\}$, we have:

- $F\left(z_{j}\right)<F(x)$, thus $z_{j} \neq x$, hence $H\left(z_{j}\right)=F\left(z_{j}\right)$; and

$-F\left(z_{j-1} \cap z_{j}\right)<F(y)$, thus $z_{j-1} \cap z_{j} \neq y$, hence $H\left(z_{j-1} \cap z_{j}\right)=F\left(z_{j-1} \cap z_{j}\right)$.

We can deduce from these two properties that $\pi^{\prime}$ is a path in $\bar{X}$ that is descending for $H$. By definition of a collapse $H(x)=H(y)$ or equivalently $H\left(x_{i-1}\right)=$ $H\left(x_{i-1} \cap z_{0}\right)$. From the underlined relations, it can be seen that:

$\pi_{1}^{\prime}=\left\langle x_{0}, \ldots, x_{i-1}, z_{0}, \ldots, z_{k}\right\rangle$ is descending for $H$. As $x \notin X$, we also infer that $\pi_{1}^{\prime}$ is a path in $\bar{X}$. Furthermore, since $\bar{X}$ is an extension of $\mathcal{M}_{F}$, the face $z_{k}$ belong to the same minimum of $F$ as $x_{\ell}$, which completes the proof.

If $X$ is a watershed of $F$, Lemmas 41 and 42 prove that we can obtain by induction a collapse $H$ of $F$ such that $X$ is a watershed of $H$ and such that the 
dimension of $\mathcal{D}_{H}$ is less than $n$. By the contraposition of Property 6 , the complex $\mathcal{D}_{H}$ does not contain any free $n$-pair. Hence, according to Definition 14, there exists a unique cut by collapse $Y$ for $\mathcal{M}_{H}$. By Theorem 15, $Y$ is also the unique cut for $\mathcal{M}_{H}$. Since $X$ is also a cut for $\mathcal{M}_{H}$, we deduce that $X=Y$. Thus, the following holds true.

Property 43 Let $X$ be a watershed of $F$, then $X$ is a cut by collapse of $F$.

\section{Conclusion}

The main notions presented in this article as well as the links between them, which constitute our main results, are synthetically presented in the diagram of Fig. 18.

The first sections (Sections 2, 3, and 4) of this paper study some subsets of a pseudomanifold of arbitrary dimension. After reminding the definitions of a complex and of a homotopic transform by collapse, we introduce the notions of an extension, a cut, and a cut by collapse particularly useful in the context of (image) segmentation. Then, a property of thinness is established for collapses and cuts (Properties 6 and 13 respectively) and the equivalence between the cuts and the cuts by collapse is proved (Theorem 15), leading to a simple and efficient algorithm to compute cuts.

The second part (Sections 5, 6, and 7) of this paper deals with maps defined on pseudomanifolds. We introduce the simplicial stacks as the maps whose level sets are simplicial complexes and we propose definitions of watersheds and of homotopic transforms by collapse for these maps. Based on the results of the first sections, we deduce an important property (Theorem 34) that links a watershed of a stack to the homotopy type of its support. This property is a generalization of a result known in a continuous setting for the particular case of distance functions. Then, the main result (Theorem 36) of this paper establishes a deep link between the watersheds and the collapses of a stack: any watershed of any stack can be straightforwardly obtained from an ultimate collapse of this stack, and conversely any ultimate collapse of the initial stack straightforwardly induces a watershed. This result, as well as Theorem 15 for the binary case, leads to efficient algorithms for computing watersheds in the framework of simplicial complexes. Additionally, we show that the collapses of maps can be used for obtaining collapses of complexes (Property 25) that satisfy an interesting properties of descending paths (Theorem 30).

The proposed framework can be applied for segmenting the triangulated surface of 3D objects (see, e.g., [44] and Fig. 6). It can also be easily transposed to cubical complexes which allows for handling digital images [6] (see an illustration to image segmentation in Fig. 7). Future work include the study of topological transformations for obtaining objects between the watersheds and the ultimate collapses of a map. These objects may be interesting for the segmentation tasks where one is interested not only by closed contours but also by pieces of curves not necessarily closed. Future work also includes the study and the proof of a property linking watersheds and collapses to the notion of minimum spanning forest known in combinatorial optimization.

\section{A Local cycles (proof of Lemma 5)}

This appendix section is devoted to a property of pseudomanifolds that allows Lemma 5 to be established. Let us first illustrate this property on an example. In Fig. 11, the set $\left\{x_{0}, \ldots, x_{7}\right\}$ is a cycle. This cycle is said "local to the point $x "$ in the sense that any of its elements belongs to $\operatorname{star}(x)$. We prove in this appendix section that there exists a cycle local to each $(n-2)$-face, in any $n$-pseudomanifold. More remarkably, Theorem 44 states, for any $(n-2)$-face $x$ of any $n$ pseudomanifold, that the set of $n$-faces of any connected component of $\operatorname{star}(x)$ is a cycle. From this result, the proof of Lemma 5 will be easily derived.

Let $X$ be a nonempty set of $n$-faces of $\mathbb{M}$. We say that $X$ is a cycle (for $\mathbb{M})$, if there exists a simple path $\pi=\left\langle x_{0}, \ldots, x_{\ell}\right\rangle$ in $\mathbb{M}$ such that $X=\left\{x_{0}, \ldots, x_{\ell}\right\}$ and such that $x_{0} \cap x_{\ell}$ is a $(n-1)$-face of $\mathbb{M}$.

Theorem 44 Let $x$ be an $(n-2)$-face of $\mathbb{M}$. The set of $n$-faces of any component of $\operatorname{star}(x)$ is a cycle of $\mathbb{M}$.

In order to prove Theorem 44, we first state Lemma 45 and Corollary 46.

By its very definition, any $k$-face (with $k \in\{0, \ldots, n\}$ ) contains $k+1$ elements. Using this fact the following result can be proved easily.

Lemma 45 Let $x$ be an $(n-2)$-face of $\mathbb{M}$, and let $x_{0}$ be an $n$-face in star $(x)$. Then, there exist exactly two distinct $(n-1)$ faces in star $(x)$ that are included in $x_{0}$.

Corollary 46 Let $x$ be an $(n-2)$-face of $\mathbb{M}$, and let $x_{1}$ be an $n$ face in star $(x)$. Then, there exist exactly two distinct $n$-faces $x_{0}$ and $x_{2}$ in star $(x)$ whose intersections with $x_{1}$ are $(n-1)$-faces.

Proof (of Theorem 44) Since any component of $\operatorname{star}(x)$ is a star, and since any nonempty star contains an $n$-face of $\mathbb{M}$ (Remark 7), to study all components of $\operatorname{star}(x)$, it is sufficient to consider, for any $n$-face $x_{0}$ in $\operatorname{star}(x)$, the component of $\operatorname{star}(x)$ that contains $x_{0}$. Let $x_{0}$ be any $n$-face in $\operatorname{star}(x)$, and let $X$ be the set of all $n$-faces of the component of $\operatorname{star}(x)$ that contains $x_{0}$.

(1) Let us first prove that $X$ includes a cycle. As a consequence of Corollary 46, we may always find $x_{1} \in X$ such that $\left\langle x_{0}, x_{1}\right\rangle$ is a simple path in $\operatorname{star}(x)$. Using again Corollary 46 , we can construct, by induction, a simple path $\pi=\left\langle x_{0}, \ldots, x_{\ell}\right\rangle$ in $\operatorname{star}(x)$ such that the only two $n$ faces in $\operatorname{star}(x)$ whose intersections with $x_{\ell}$ are $(n-1)$ faces of $\mathbb{M}$ both belong to $\left\{x_{0}, \ldots x_{\ell}\right\}$. By construction, one of these two $n$-faces is $x_{\ell-1}$. Let us denote the other one 


\begin{tabular}{l||l|l|l|l|}
$\begin{array}{l}\text { Notions defined } \\
\text { for a simplicial } \\
\text { complex } \bar{A} \text {. }\end{array}$ \\
$\begin{array}{l}\text { Properties } \\
\text { that hold true } A=\mathcal{M}_{F}\end{array}$
\end{tabular}

Fig. 18 A diagram summarizing the main results established in this paper.

by $z$. Necessarily $z=x_{i}$, for some $i \in\{0, \ldots, \ell-2\}$. If $i>0$, then $x_{i-1}, x_{i+1}$ and $x_{\ell}$ are three distinct faces in $\operatorname{star}(x)$ whose intersections with $x_{i}$ are $(n-1)$-faces of $\mathbb{M}$, which constitutes a contradiction with Corollary 46. Thus, we necessarily have $z=x_{0}$. Hence, the set $\left\{x_{0}, \ldots, x_{\ell}\right\} \subseteq X$ is a cycle.

(2) Let us now prove, by contradiction, that $X=\left\{x_{0}, \ldots, x_{\ell}\right\}$, hence, by (1), that $X$ is a cycle. Suppose that there exists an element $z$ in $X$ such that $z \notin\left\{x_{0}, \ldots, x_{\ell}\right\}$. By definition of $X$, there exists, in $\operatorname{star}(x)$, a simple path $\pi^{\prime}=\left\langle y_{0}, \ldots y_{m}\right\rangle$ from $x_{0}=y_{0}$ to $z=y_{m}$. Let $k \in$ $\{1, \ldots, m\}$ be the lowest index such that $y_{k} \notin\left\{x_{0}, \ldots, x_{\ell}\right\}$. Hence, $y_{k-1} \in\left\{x_{0}, \ldots, x_{\ell}\right\}$. By Corollary 46, there exist exactly two $n$-faces in $\operatorname{star}(x)$, whose intersections with $y_{k-1}$ are $(n-1)$-faces of $\mathbb{M}$. By construction of $\pi$, these two $n$-faces belong to $\left\{x_{0}, \ldots, x_{\ell}\right\}$. Hence, we have $y_{k} \in\left\{x_{0}, \ldots, x_{\ell}\right\}$, a contradiction. Thus, since by (1), we have $\left\{x_{0}, \ldots, x_{\ell}\right\} \subseteq X$, we deduce that $X=$ $\left\{x_{0}, \ldots, x_{\ell}\right\}$.

Theorem 44 can be easily verified on Fig. 11. It can also be verified on the 2-pseudomanifold shown in Fig. 10b. In particular, let $x$ denote the 0 -face represented by a light gray dot. It can be seen that $\operatorname{star}(x)$ includes two components: one is made of the triangles and edges at the left of $x$, and the other is made of the triangles and edges at the right of $x$. It can be easily seen that the sets of triangles associated to this two components are cycles for the considered 2pseudomanifold.

Remark also that, if $\mathbb{M}$ is a not a pseudomanifold, then Theorem 44 is, in general, not true. For instance, let us consider the complex of Fig. 9c, which is not a pseudomanifold, and let $x$ be any of the two points that belong to the edge depicted in light gray (i.e., the pinching). The set $\operatorname{star}(x)$ itself is the only component of $\operatorname{star}(x)$. However, it can be verified that the set of all triangles in $\operatorname{star}(x)$ is not a cycle.

Proof (of Lemma 5) Clearly, the two $n$-faces $x_{0}$ and $x_{1}$ belong to the same component $X$ of $\operatorname{star}(x)$. The set $X_{n}$ of all $n$ faces of $X$ is, by Theorem 44, a cycle. Thus, as $x_{0}$ and $x_{1}$ belong to $X$, it can be seen that there exists, in $\operatorname{star}(x)$, two distinct simple paths $\pi=\left\langle y_{0}=x_{0}, \ldots, y_{\ell}=x_{1}\right\rangle$ and $\pi^{\prime}=\left\langle z_{0}=x_{0}, \ldots, z_{m}=x_{1}\right\rangle$ from $x_{0}$ to $x_{1}$ such that $\left\{y_{1}, \ldots, x_{\ell}\right\} \cap\left\{z_{1}, \ldots, z_{m-1}\right\}=\emptyset$. At least one of $\pi$ and $\pi^{\prime}$ is a path in $\operatorname{star}(X) \backslash\{y\}$. Therefore, $x_{0}$ and $x_{1}$ are linked for $\operatorname{star}(x) \backslash\{y\}$.

\section{References}

1. Arcelli, C., Di Baja, G.S.: A width-independent fast thinning algorithm. IEEE Transactions on Pattern Analysis and Machine Intelligence 7(4), 463 -474 (1985)

2. Attene, M., Katz, S., Mortara, M., Patane, G., Spagnuolo, M., Tal, A.: Mesh segmentation - a comparative study. In: Proceedings of the IEEE International Conference on Shape Modeling and Applications 2006, SMI '06, p. 7 pages (2006)

3. Bertrand, G.: On topological watersheds. Journal of Mathematical Imaging and Vision 22(2-3), 217-230 (2005)

4. Bertrand, G.: On critical kernels. Comptes Rendus de l'Académie des Sciences, Série Math. I 345, 363-367 (2007)

5. Bertrand, G., Couprie, M.: Two-dimensional parallel thinning algorithms based on critical kernels. Journal of Mathematical Imaging and Vision 31(1), 35-56 (2008)

6. Bertrand, G., Couprie, M., Cousty, J., Najman, L.: Chapter 3 - Watersheds in discrete spaces. In: T.H. Najman Laurent (ed.) Mathematical morphology: from theory to applications, pp. 81-107. ISTE-Wiley (2010)

7. Bertrand, G., Everat, J.C., Couprie, M.: Image segmentation through operators based upon topology. Journal of Electronic Imaging 6(4), 395-405 (1997)

8. Beucher, S., Meyer, F.: The morphological approach to segmentation: the watershed transformation. E. Dougherty (Ed.), Mathematical Morphology in Image Processing, Marcel Decker pp. 443-481 (1993)

9. Blum, H.: An associative machine for dealing with the visual field and some of its biological implications. In: E.E. Bernard, M.R. Kare (eds.) 2nd Annual Bionics Symposium. Cornell University, Plenum Press (1961)

10. Blum, H.: A transformation for extracting descriptors of shape. In: Models for the Perception of Speech and Visual Forms, pp. 362-380. MIT Press (1967)

11. Boussinesq, J.: Essai sur la théorie des eaux courantes. Mémoires présentés par divers savants à l'Académie des Sciences pp. 162-178 (1972). Chapter Disgression sur les thalwegs et les faîtes à la surface du sol et sur leur 
rapports avec les lignes de déclivités minima, Institut de France

12. Cardoso, M., Clarkson, M., Modat, M., Ourselin, S.: Longitudinal cortical thickness estimation using Khalimsky's cubic complex. In: G. Fichtinger, A. Martel, T. Peters (eds.) Medical Image Computing and Computer-Assisted Intervention - MICCAI 2011, Lecture Notes in Computer Science, vol. 6892, pp. 467-475. Springer Berlin / Heidelberg (2011)

13. Cardoso, M., Clarkson, M., Modat, M., Ourselin, S.: On the extraction of topologically correct thickness measurements using Khalimsky's cubic complex. In: G. Székely, H. Hahn (eds.) Information Processing in Medical Imaging, Lecture Notes in Computer Science, vol. 6801, pp. 159-170. Springer (2011)

14. Cayley, A.: On contour and slope lines. Philosophical Magazine Series 4 18, 264-268 (1859)

15. Chaussard, J., Couprie, M.: Surface thinning in 3d cubical complexes. In: P. Wiederhold, R.P. Barneva (eds.) Combinatorial Image Analysis, 13th International Workshop, IWCIA 2009. Proceedings, Lecture Notes in Computer Science, vol. 5852, pp. 135-148. Springer (2009)

16. Comic, L., Mesmoudi, M.M., Floriani, L.D.: Smalelike decomposition and Forman theory for discrete scalar fields. In: I. Debled-Rennesson et al. (ed.) Discrete geometry for computer imagery, Lecture Notes in Computer Science, vol. 6607, pp. 477-488. Springer (2011)

17. Cormen, T.H., Leiserson, C.E., Rivest, R.L., Stein, C.: Introduction to Algorithms, 2 edn. The MIT Press (2001)

18. Couprie, M., Bertrand, G.: Topological grayscale watershed transform. In: Procs. of SPIE Vision Geometry V, vol. 3168, pp. 136-146 (1997)

19. Couprie, M., Bertrand, G.: New characterizations of simple points in $2 \mathrm{D}, 3 \mathrm{D}$, and $4 \mathrm{D}$ discrete spaces. IEEE Transactions on Pattern Analysis and Machine Intelligence 31(4), 637-648 (2009)

20. Couprie, M., Bezerra, F.N., Bertrand, G.: Topological operators for grayscale image processing. Journal of Electronic Imaging 10(4), 1003-1015 (2001)

21. Cousty, J., Bertrand, G., Couprie, M., Najman, L.: Fusion graphs: merging properties and watersheds. Journal of Mathematical Imaging and Vision 30(1), 87-104 (2008)

22. Cousty, J., Bertrand, G., Couprie, M., Najman, L.: Collapses and watersheds in pseudomanifolds. In: P. Wiederhold, R.P. Barneva (eds.) Combinatorial Image Analysis, 13th International Workshop, IWCIA 2009. Proceedings, Lecture Notes in Computer Science, vol. 5852, pp. 397-410. Springer (2009)

23. Cousty, J., Bertrand, G., Najman, L., Couprie, M.: Watershed cuts: Minimum spanning forests and the drop of water principle. IEEE Transactions on Pattern Analysis and Machine Intelligence 31(8), 1362-1374 (2009)

24. Cousty, J., Bertrand, G., Najman, L., Couprie, M.: Watershed cuts: thinnings, shortest-path forests and topological watersheds. IEEE Transactions on Pattern Analysis and Machine Intelligence 32(5), 925-939 (2010)

25. Cousty, J., Najman, L., Bertrand, G., Couprie, M.: Weighted fusion graphs: merging properties and watersheds. DAM 156(15), 3011-3027 (2008)

26. Digabel, H., Lantuéjoul, C.: Iterative algorithms. In: 2nd European Symp. Quantitative Analysis of Microstructures in Material Science, Biology and Medicine, pp. 8589 (1978)
27. Edelsbrunner, H., Harer, J.: The persistent Morse complex segmentation of a 3-manifold. In: N. MagnenatThalmann (ed.) Modelling the Physiological Human, Lecture Notes in Computer Science, vol. 5903, pp. 36-50. Springer (2009)

28. Floriani, L.D., Mesmoudi, M.M., Danovaro, E.: A smalelike decomposition for discrete scalar fields. In: Pattern Recognition, 2002. Proceedings. 16th ICPR, pp. 184-187 (2002)

29. Jerse, G., Kosta, N.M.: Ascending and descending regions of a discrete Morse function. Computational Geometry 42(6-7), 639 - 651 (2009)

30. Jordan, C.: Nouvelles observations sur les lignes de faîtes et de thalweg. Comptes Rendus des Séances de l'Académie des Sciences 75, 1023-1025 (1872)

31. Kong, T., Rosenfeld, A.: Digital topology: Introduction and survey. Computer Vision, Graphics, and Image Processing 48(3), 357-393 (1989)

32. Kong, T.Y.: Topology-preserving deletion of 1's from 2-, 3- and 4-dimensional binary images. In: E. Ahronovitz, C. Fiorio (eds.) Discrete geometry for computer imagery, Lecture Notes in Computer Science, vol. 1347, pp. 3-18. Springer (1997)

33. Kovalevsky, V.A.: Finite topology as applied to image analysis. Computer Vision, Graphics, and Image Processing 46(2), 141-161 (1989)

34. Lavoué, G., Vandeborre, J.P., Benhabiles, H., Daoudi, M., Huebner, K., Mortara, M., Spagnuolo, M.: SHREC'12 Track: 3D mesh segmentation. In: Eurographics 2012 Workshop on 3D Object Retrieval, pp. 93-99. Cagliari, Italie (2012)

35. Lieutier, A.: Any open bounded subset of $\mathrm{R}^{\mathrm{n}}$ has the same homotopy type as its medial axis. Computer-Aided Design 36(11), 1029-1046 (2004)

36. Liu, L., Chambers, E.W., Letscher, D., Ju, T.: A simple and robust thinning algorithm on cell complexes. Computer Graphics Forum 29(7), 2253-2260 (2010)

37. Mangan, A.P., Whitaker, R.T.: Partitioning 3d surface meshes using watershed segmentation. IEEE Transactions on Visualization and Computer Graphics 5(4), 308$321(1999)$

38. Matheron, G.: Quelques propriétés topologiques du squelette (1978). URL http://cg.ensmp.fr/bibliotheque/ public/MATHERON_Rapport_00213.pdf. Rapport interne du centre de géoostatistique de l'Ecole Nationale Supérieure des Mines de Paris

39. Matheron, G.: Examples of Topological Properties of Skeletons. In: J. Serra (ed.) Image analysis and mathematical morphology. Volume 2: theoretical advances, chap. 11, pp. 217-238. Academic Press (1988)

40. Maunder, C.R.F.: Algebraic Topology. Dover, New-York (1996)

41. Maxwell, J.: On hills and dales. Philosophical Magazine 4/40, 421-427 (1870)

42. Najman, L., Schmitt, M.: Watershed of a continuous function. Signal Processing 38(1), 68-86 (1993)

43. Noël, L., Chaussard, J., Biri, V.: Coarse irradiance estimation using curvilinear skeleton. In: ACM SIGGRAPH 2012 Posters, pp. 101:1-101:1. ACM (2012)

44. Philipp-Foliguet, S., Jordan, M., Najman, L., Cousty, J.: Artwork 3d model database indexing and classification. Pattern Recognition 44(3), 588-597 (2011)

45. Ranwez, V., Soille, P.: Order independent homotopic thinning for binary and grey tone anchored skeletons. Pattern Recognition Letters 23(6), 687 - 702 (2002)

46. Rivière, A.: Classification des points d'un ouvert d'un espace euclidien relativement à la distance au bord: 
Etude topologique et quantitative des classes obtenues. Ph.D. thesis, Université de Paris-sud, centre d'Orsay (1987)

47. Rivière, A.: Nervure d'un Ouvert d'un Espace Euclidien. J. Sci. Univ. Tehran (Sec. A: Math) 1, 1-24 (1996)

48. Robins, V., Wood, P.J., Sheppard, A.P.: Theory and algorithms for constructing discrete Morse complexes from grayscale digital images. IEEE Transactions on Pattern Analysis and Machine Intelligence 33(8), 16461658 (2011)

49. Roerdink, J.B.T.M., Meijster, A.: The watershed transform: Definitions, algorithms and parallelization strategies. Fundamenta Informaticae 41(1-2), 187-228 (2001)

50. Ronse, C.: Partial partitions, partial connections and connective segmentation. Journal of Mathematical Imaging and Vision 32(2), 97-125 (2008)

51. de Saint-Venant, M.: Surfaces à plus grande pente constituées sur des lignes courbes. Bulletin de la Soc. Philomath. de Paris pp. 24-30 (1852)

52. Saùde, A.V., Couprie, M., Lotufo, R.A.: Discrete 2D and $3 \mathrm{D}$ euclidean medial axis in higher resolution. Image and Vision Computing 27(4), 354 - 363 (2009)

53. Serra, J.: image analysis and mathematical morphology, volume 1. Academic press (1982)

54. Shamir, A.: A survey on mesh segmentation techniques. Computer Graphics Forum 27(6), 1539-1556 (2008)

55. Vincent, L., Soille, P.: Watersheds in digital spaces: An efficient algorithm based on immersion simulations. IEEE Transactions on Pattern Analysis and Machine Intelligence 13(6), 583-598 (1991)

56. Whitehead, J.H.C.: Simplicial Spaces, Nuclei and mGroups. Proc. London Math. Soc. s2-45(1), 243-327 (1939) 